\title{
Das „Transformative Projektseminar“ - didaktische Ansätze und methodische Umsetzung
}

\author{
Richard Beecroft
}

\section{Anwendungskontext}

\subsection{Der Erfahrungshintergrund}

Die hier vorgestellte Methodik des „Transformativen Projektseminars“ (TraPS) basiert auf Erfahrungen der „Karlsruher Schule der Nachhaltigkeit“ (KSN), von der sechs TraPS in einem Zeitraum von drei Jahren durchgeführt wurden. Das Konzept des TraPS wurde in der Zeit iterativ weiterentwickelt, die hier vorgestellte Form stellt einen Idealtyp auf Basis dieser Erfahrungen dar. Die sechs Kurse im Einzelnen und wesentliche Anpassungsschritte in der Struktur sind im Anhang zu diesem Beitrag dargestellt.

Eine Besonderheit war die Einbettung dieser sechs TraPS in ein Reallabor (im Folgenden als „Reallabor Karlsruhe“ bezeichnet, s. auch Steckbrief im Anhang zu diesem Buch): Es setzt sich zusammen aus mehreren Projekten, beginnend im Jahr 2012 mit dem „Quartier Zukunft - Labor Stadt“. Mit dem Projekt „Reallabor 131: KIT findet Stadt“" (2015-2017, 2018-2019) wurden die Aktivitäten das erste Mal explizit als Reallabor gerahmt. Gemeinsamer Zielhorizont der Aktivitäten war die Nachhaltigkeitstransformation eines Karlsruher Stadtteils, der Oststadt (s. Waitz et al. 2018; Trenks et al. 2018; Parodi et al. 2016; Beecroft und Parodi 2016; Meyer-Soylu et al. 2016). Wichtig in der Architektur des Reallabors ist eine Trennung zwischen a) dem Labor als Infrastruktur, b) übergreifenden Aufgaben des Laborbetriebs und c) den transdisziplinären Projekten, die darin stattfinden (Beecroft et al. 2018). Neben Realexperimenten und kleineren partizipativen Veranstaltungsformaten waren dies im Reallabor Karlsruhe u. a. die TraPS.

Ein Kernelement der Infrastruktur ist der seit 2015 bestehende „Zukunftsraum für Nachhaltigkeit und Wissenschaft", ein multifunktionaler Projektraum mitten im Stadtquartier, der als Büro, Treffpunkt, Lernumgebung und Eventraum genutzt 
wird, sowohl vom Forschungsteam des Reallabors selbst als auch von unterschiedlichen Praxispartnern des Reallabors, insbesondere zivilgesellschaftlichen Gruppen. Die Wechselwirkung der TraPS mit dem Reallabor Karlsruhe wurde anhand der sechs bislang durchgeführten TraPS untersucht (Beecroft 2018), siehe auch Abschnitt 5.3.

Die Einbettung der TraPS im Reallabor stellte eine Einbettung in die Forschung dar, die die Potenziale für Nachhaltige Entwicklung auf Quartiersebene, insbesondere auch in kultureller und sozialer Hinsicht, untersuchte. Das Reallabor hatte auch ein deutliches Profil in der Kooperation mit zivilgesellschaftlichen Initiativen im Quartier und der Stadt Karlsruhe, so dass die Praxiseinbettung der TraPS in der Regel durch die Kooperation mit den jeweiligen Praxispartnern gegeben war. Die Lehreinbettung der TraPS lag beim Zentrum für Angewandte Kulturwissenschaft und Studium Generale (ZAK) am Karlsruher Institut für Technologie. Das ZAK bot u. a. ein freiwilliges „Begleitstudium Nachhaltige Entwicklung" für alle Student(inn)en an. Dieses wurde gemeinsam mit der KSN konzipiert und umfasste neben einem Einführungs- und einem Wahlpflichtmodul auch ein transdisziplinäres Projektmodul, für das insbesondere ein TraPS belegt werden kann.

Das TraPS ist keine grundsätzlich neue Lehrform, sondern integriert mehrere Ansätze aus dem State of the Art fachübergreifender, aktiver Lehre mit einer Reihe ausgewählter methodisch-didaktischer Elemente $\mathrm{zu}$ einer transdisziplinären Arbeitsweise mit transformativem Anspruch. In den folgenden Abschnitten (1.2-1.4) werden zunächst geeignete Anwendungsfälle für ein TraPS, die Akteurskonstellation und Voraussetzungen benannt. In Kapitel 2 wird die Phasenstruktur des TraPS beschrieben. In Kapitel 3 werden die didaktischen Ansätze, die der TraPS-Konzeption zu Grunde liegen, vorgestellt und in Kapitel 4 um didaktische Einzelmethoden und Themen ergänzt, die für die Umsetzung eines TraPS besonders zu beachten sind. Kapitel 5 reflektiert die Erfahrungen mit TraPS anhand einiger zentraler Themen.

\subsection{Wann eignet sich das Transformative Projektseminar?}

Das TraPS ist ein Lehrformat, um parallel Bildungs-, Praxis- und Forschungsziele zu verfolgen. Es verbindet mehrere didaktische Ansätze zu einem transdisziplinären Prozess, der zeitlich in sechs Phasen strukturiert ist.

Bildungs-, Praxis- und Forschungsziele beschreiben zunächst nur die Zieldimensionen, um die es geht. Welche spezifischen Ziele in den einzelnen Dimensionen verfolgt werden, muss für jedes TraPS neu festgelegt werden: Ein TraPS kann im Bereich der Praxis dazu eingesetzt werden, Praxispartner eines Reallabors oder eines vergleichbaren Forschungsprojekts mit einer Reihe von kleineren Maßnah- 
men zu unterstützen. Dies bietet sich zum Beispiel an bei vielschichtigem oder diffusem Bedarf, um umfassendere Transformationsprozesse zu unterstützen, sowie indirekt, um langfristige Kooperationen anzubahnen, auch zwischen Praxispartnern. Es dient explizit nicht dazu, als Einzelmaßnahme Transformationsprozesse zu initiieren, sondern dazu, existierende zu verstärken.

Im Bereich der Bildung dient es dazu, verantwortliche Eigenaktivitäten und Reflexionsvermögen der Student(inn)en zu fördern, zum Beispiel gerahmt als Bildung für Nachhaltige Entwicklung oder Bildung durch Engagement. Darüber hinaus ist möglich, dass Praxispartner und Forschungspartner im Projekt auch eigene Bildungsziele verfolgen. Zumindest implizite Bildungswirkungen sind für alle Partner zu erwarten.

Im Hinblick auf die Forschung bietet das TraPS die Möglichkeit, erstens ein neues Feld zu erschließen, zweitens eine mehrdimensionale Problemanalyse und Lösungssuche durchzuführen (z. B. unter verschiedenen Nachhaltigkeitsgesichtspunkten) sowie drittens kleinskalige, eher risikoarme Interventionen durchzuführen und zu dokumentieren. Unter Forschungsgesichtspunkten ist das TraPS gut an unterschiedliche wissenschaftliche Kontexte adaptierbar, um beispielsweise unterschiedlichen disziplinären Anforderungen zu entsprechen, bestimmte Modelle oder Theorien zu verwenden oder einen bestimmten Gegenstand in den Mittelpunkt zu stellen. In jedem Fall bietet es einen Anlass zur kontinuierlichen interdisziplinären Kooperation. Die Transformationswirkung des TraPS basiert nicht auf einer großen Intervention, sondern auf mehreren kleineren Beiträgen, die parallel von Studierendenteams erarbeitet werden, aus denen sich in der praktischen Anknüpfung einzelne als zentral erweisen können, während andere keine weitere Dynamik entfalten.

Im Rahmen größer angelegter transdisziplinärer Projekte ist das TraPS insbesondere geeignet für eine Phase, in der noch Kooperationen stabilisiert werden sollen, aber schon erste Interventionen beginnen. Aufgrund seiner dreifachen Zielsetzung ist das TraPS recht flexibel einsetzbar.

Ein Transfer in andere Bildungskontexte (eher berufliche und Weiterbildung als Schulbildung) ist denkbar. Eine regelmäßige Anwendung mit denselben Partnern ist denkbar, falls im Projekt Praxispartner mit regelmäßig neuen Bedarfen beteiligt werden können. Das TraPS kann im Prinzip über mehrere Semester gestreckt, allerdings nicht wesentlich gekürzt werden.

Die wichtigste Einschränkung eines TraPS liegt darin begründet, dass die Freiheit der Student(inn)en im Sinne des forschenden Lernens (s. Abschnitt 3.2) in Konkurrenz dazu geraten kann, die im TraPS zu erarbeitenden Ergebnisse im Voraus zu planen. Das TraPS steht und fällt mit einer Gruppe engagierter Student(inn)en. Wenn nur geringe Aussichten bestehen, Student(inn)en zu gewinnen, beispiels- 
weise wegen fehlender Anerkennung, unattraktivem Thema oder zu vielen vergleichbaren Kursen, sollte auf das Format eher verzichtet werden. Es ist nicht geeignet für ein Lehrteam ohne jede Lehrerfahrung in projektförmiger Lehre.

\subsection{Voraussetzungen}

Voraussetzungen für die Durchführung eines TraPS sind:

1) Praxispartner mit Anliegen, die sich innerhalb eines Semesters bearbeiten lassen. Solche Anliegen der Praxispartner dürfen heterogen und unspezifisch sein. Im Rahmen der Karlsruher TraPS waren insbesondere kleine zivilgesellschaftliche Gruppen mit Nachhaltigkeitsbezug beteiligt, die bestimmte Aktivitäten nicht aus eigener Kraft bewältigen konnten.

2) Student(inn)en, die im Rahmen ihres Studiums oder freiwillig am TraPS teilnehmen können. Es ist ideal, wenn sie aus unterschiedlichen Fachrichtungen, aber mit einem gewissen gemeinsamen Grundwissen teilnehmen (im Fall der Karlsruher TraPS nahmen viele Student(inn)en des freiwilligen Begleitstudiums Nachhaltige Entwicklung Teil, die das TraPS in der Regel erst nach einigen einführenden Kursen besuchten). Für andere Studierendengruppen muss die Struktur gegebenenfalls angepasst werden.

3) Ein wissenschaftlicher Projektrahmen, der Impulse aus dem Seminar inhaltlich aufgreifen kann. Im Karlsruher Fall war dies das Reallabor. Andere, längerfristige Rahmen (Fachbereiche, Arbeitsstellen, Forschungsprojekte mit hinreichend langer Laufzeit, Wissenschaftsläden, städtische Wissenschaftsbüros etc.) bieten ein ähnliches Potenzial. Wichtig ist, dass Ressourcen auch für die Zeit nach dem Seminar vorgesehen sind, im Einzelfall kann dies auch ein Praxispartner übernehmen.

Wenn eine der drei Bedingungen nur knapp erfüllt ist, kann ein TraPS (leicht angepasst) durchgeführt werden, wenn hingegen mehrere Bedingungen nicht solide gegeben sind, ist die Sinnhaftigkeit dieser Arbeitsweise überaus fraglich. ${ }^{1}$

1 Die in Kapitel 3 dargestellten didaktischen Ansätze bieten ggf. eine Auswahl an anderen Zugängen, die jeweils spezialisierter eine der Zielsetzungen in den Mittelpunkt rücken. 


\subsection{Beteiligte Akteure und deren Rollen}

Das TraPS beteiligt im Kern drei Gruppen in jeweils mehreren Rollen:

1) Wissenschaftler(innen), die im wissenschaftlichen Projektrahmen tätig sind, wenn möglich als Lehrteam aus zwei Personen. Von diesen geht zumeist die Initiative für das TraPS aus, sie beteiligen die anderen Partner.

2) Student(inn)en als Seminarteilnehmer(innen), ideal 12 bis 24 Personen, sowie evtl. als Hilfskräfte. Die Student(inn)en arbeiten in den Phasen III bis V in Teams zusammen, in den Phasen II und VI sind sie eher als Einzelpersonen beteiligt.

3) Praxispartner, die am Projekt beteiligt sind, wie Mitglieder von zivilgesellschaftlichen Gruppen (Vereine, Kirchengemeinden, Initiativen), Mitarbeiter(innen) aus kommunalen Einrichtungen (Agendabüros, Schulen, Ämter, Kultureinrichtungen) oder Unternehmen (sowie z. B. Branchenverbände, Kammern). Es sollten idealerweise 2 bis 3 Personen beteiligt sein.

Ob die Motivation, ein TraPS durchzuführen, in der Lehrplanung durch Wissenschaftler(innen), in einem Forschungsprojekt mit transformativem Anspruch oder in einem praktischen Anliegen gründet, ist unerheblich, solange letztendlich die Einbettung in Bildungs-, Forschungs- und Praxiskontexte möglich ist und die oben genannten Voraussetzungen gegeben sind. Die Erfahrung mit zivilgesellschaftlichen Gruppen war im Reallabor Karlsruhe besonders gut, was zumindest teilweise am Elan der dort Engagierten lag. Gegebenenfalls kann auch mehr als eine Gruppe von Praxispartnern beteiligt werden, sofern die Themenstellung dies rechtfertigt und die Rollen klar sind. Bei einem Karlsruher TraPS, in dem ein „Nachhaltigkeitsspaziergang“ entwickelt wurde, waren beispielsweise Vertreter(innen) der Stadtverwaltung - primär als Expert(inn)en - und eines eingetragenen Vereins, der alternative Stadttouren anbietet - als Expert(inn)en und Adressat(inn)en -, beteiligt. Die Phasenstruktur erlaubt es zudem, relativ leicht weitere Wissenschaftler(innen) in geringem Umfang zu involvieren, beispielsweise durch Gastvorträge oder als Interviewpartner(innen). Damit ergibt sich die Akteurskonstellation in Abb. 1, dargestellt für die Phasen III bis V, in denen die Student(inn)en in Teams zusammenarbeiten.

Man darf aus dieser Konstellation nicht die Schlussfolgerung ziehen, die drei Gruppen hätten jeweils nur eine der drei Zieldimensionen im Auge. Es ist keineswegs so, dass Student(inn)en nur zum Lernen, Praxispartner nur für die Förderung ihrer Praxis und Wissenschaftler(innen) nur für die Forschung stehen - dann wäre ein gemeinsamer Prozess auch schwer umzusetzen. Vielmehr nehmen Personen aus allen drei Gruppen bezogen auf diese Zieldimensionen unterschiedliche Rollen ein. 


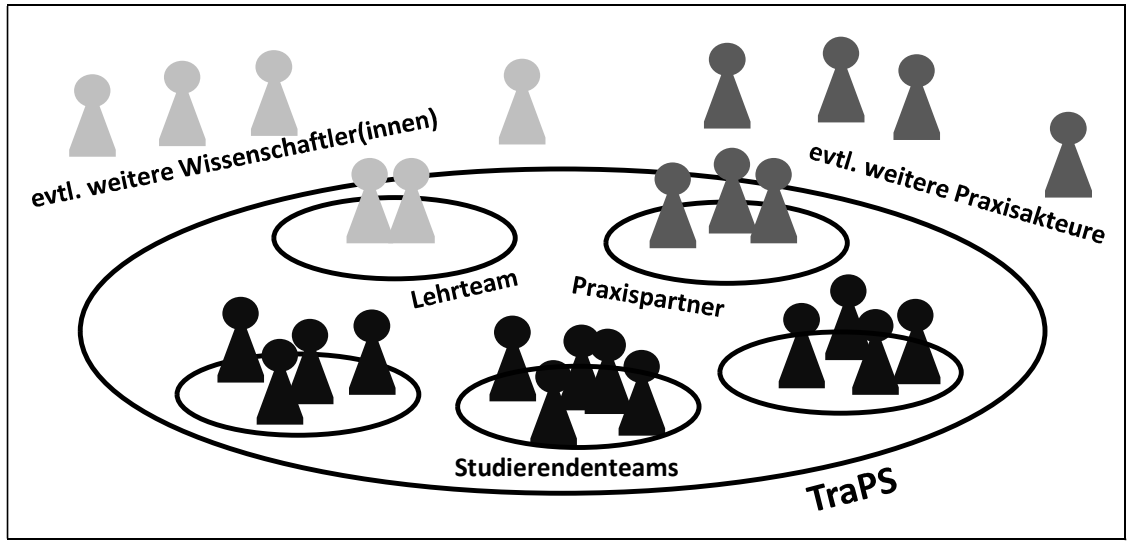

Abbildung 1: Akteurskonstellation im Transformativen Projektseminar (Phasen III-V). (C) Richard Beecroft.

- Student(inn)en können zum Beispiel ihre Rolle als Lernende verbinden mit denen als Change Agents - also als Vorreiter(innen) oder aktive Treiber(innen) von Transformationsprozessen -, als Forschende, die recherchieren, experimentieren, auswerten und abstrahieren, als kritische Beobachter(innen), als Vermittler(innen), als Betroffene oder als politisch interessierte Bürger(innen).

- Den Lehrenden kommen schon klassischerweise verschiedene Rollen zu: Als Expert(inn)en für Methoden und Gegenstände, als Leitung mit Verantwortung wie für den Lernort und die Zeitplanung, als Beobachter(innen) der Lernprozesse, als Bewerter(innen) der Leistungen der Student(inn)en. In transdisziplinären Settings kommen weitere Rollen hinzu, insbesondere als Moderator(inn)en von Gruppenprozessen, als Gesprächspartner(innen) zur Reflexion, als Mediator(inn)en bei Konflikten, als wissenschaftliche Partner gegenüber den Praxispartnern, als Vermittler(innen) von Kontakten zu weiteren Akteuren, als Lernende und als Evaluator(inn)en.

- Die Praxispartner sind auf jeden Fall als Expert(inn)en für ihr jeweiliges Themenfeld und als Adressat(inn)en für das Ergebnis der Student(inn)en beteiligt, aber andere Rollen umfassen die als Advokat(inn)en für ihr Anliegen, als Gesprächspartner(innen) für die Student(inn)en, als Mitbewerter(innen) der studentischen Leistungen, als Werber(innen) für ihre Initiativen, als Vermittler(innen) von weiteren Kontakten und als Lernende.

Durch die Gleichzeitigkeit von Forschungs-, Bildungs- und Praxiszielen, durch die gezielt herbeigeführten Erfahrungen mit Neuem und die intensive Zusammenarbeit werden immer wieder Rollenverschiebungen nötig. Einige Rollen hängen 
von der jeweiligen Phase des TraPS ab. Die Lehrenden treten etwa tendenziell am Anfang vor allem als Leiter(innen) und Expert(inn)en, während der Projektarbeit vor allem als Moderator(inn)en, Gesprächspartner(innen) und Beobachter(innen), zum Schluss als Bewerter(innen) und als Ansprechpartner(innen) für die Praxispartner in Erscheinung. Sowohl eingangs, bei der Formulierung der Bedarfe, als auch abschließend bei der Übergabe von Resultaten kommt es oft zu Rollenwechseln. Andere Rollen, wie die Rolle der Lehrenden als Verantwortliche für den Rahmen, bleiben durchgängig relevant. Eine Form der Rollenverschiebung, die sich in den sechs TraPS in Karlsruhe zeigte, waren Rollenwechsel zwischen den drei Gruppen, zum Beispiel:

- Praxispartner wurden zu Co-Lehrenden.

- Student(inn)en wurden zu Mitgliedern der Initiativen, die als Praxispartner beteiligt waren.

- Student(inn)en waren Mitglieder anderer zivilgesellschaftlicher Gruppen, die als zusätzliche Praxispartner einbezogen wurden.

Neben Rollenverschiebungen kommt es im TraPS immer wieder auch zu Rollenüberschneidungen. Wie in vielen anderen lebensweltlichen Situationen decken sich die Erwartungen der anderen beteiligten Personen an einen nicht immer mit der je eigenen Rollenvorstellung, und auch jede(r) einzelne Beteiligte(r) kann in ihrem/seinem Selbstverständnis zwischen mehreren Rollenvorstellungen schwanken. Problematisch werden solche Rollenüberschneidungen aber erst dann, wenn die Rollen nicht vereinbare Aktivitäten erforderlich machen. Weiterhin können Rollen außerhalb des TraPS-Kontextes in die Rollenvielfalt hineinspielen. Eine Praxispartnerin mit sozialwissenschaftlichem Beruf freute sich beispielsweise zu Beginn eines Karlsruher TraPS mit ungefähr diesen Worten: „Cool, jetzt bin ich Praxispartnerin - sonst habe ich immer welche!“ Ein typischer Rollenkonflikt kann auch zwischen beruflichem und ehrenamtlichem Engagement der Beteiligten aufbrechen.

Sowohl Rollenverschiebungen als auch Rollenüberschneidungen sind nicht per se problematisch, sollten aber gegebenenfalls explizit angesprochen werden, um unangemessene Erwartungen aneinander zu vermeiden - oft reicht hierzu ein kurzer Hinweis („Als Wissenschaftler(in) finde ich diesen Punkt sehr spannend, als Lehrende(r) muss ich Sie aber an Ihren Zeitplan erinnern ..."). In der Reflexion des Lehrteams zwischen den Terminen sollten Rollenkonflikte oder unerwünschte Rollenverschiebungen jeweils thematisiert werden. 


\section{Idealtypischer Ablauf eines Transformativen Projektseminars}

Ein TraPS folgt einem Ablaufschema von sechs Phasen, die jeweils unterschiedlichen Zielen dienen und in denen unterschiedliche Arbeitsweisen zum Einsatz kommen (s. Abb. 2).

Phasen II bis V entsprechen einem normalen Semestertakt mit 14 wöchentlichen Terminen à 90 Minuten und eigenständiger Arbeit dazwischen. Phasen I und VI rahmen die Seminarphasen ein; sie dienen insbesondere dem transdisziplinären Charakter des TraPS. Ihre Länge kann nicht allgemein beschrieben werden, da sowohl die Anbahnung einer Kooperation als auch die In-Wert-Setzung der Ergebnisse und Produkte wesentlich von bestehenden Kooperationsbeziehungen abhängen. Bei der Anbahnung sollte immer die Möglichkeit mitgedacht werden, dass keine Kooperation zustande kommt - zumindest nicht in Form eines TraPS.

Abb. 2 verdeutlicht auch die Bedeutung und den Aufwand der Arbeitsschritte zwischen den Seminarsitzungen für die Student(inn)en. Die Zeile, die mit „PP“ markiert ist, zeigt, an welchen Terminen die Praxispartner unbedingt $(++)$, eher $(+)$ oder eher nicht $(-)$ anwesend sein sollten, In der Zeile „St.“ ist ausgewiesen, wann die Student(inn)en in parallelen Teams arbeiten.

Im Folgenden werden für jede der Phasen die idealtypisch aufeinander folgenden Arbeitsschritte benannt. Zwischen den Terminen ist es ratsam, dass sich das Lehrteam zumindest kurz darüber austauscht, welche Entwicklungen in der Seminargruppe als Ganzes, in den einzelnen Teams, gegebenenfalls bei einzelnen Studierenden sowie in der Zusammenarbeit mit den Praxispartnern aufgefallen sind sowohl auf der Ebene der Gruppendynamik als auch auf der sachlichen Ebene. In diesen Reflexionsgesprächen muss abgewogen werden, ob eine Intervention oder ein inhaltlicher oder methodischer Input nötig ist. 


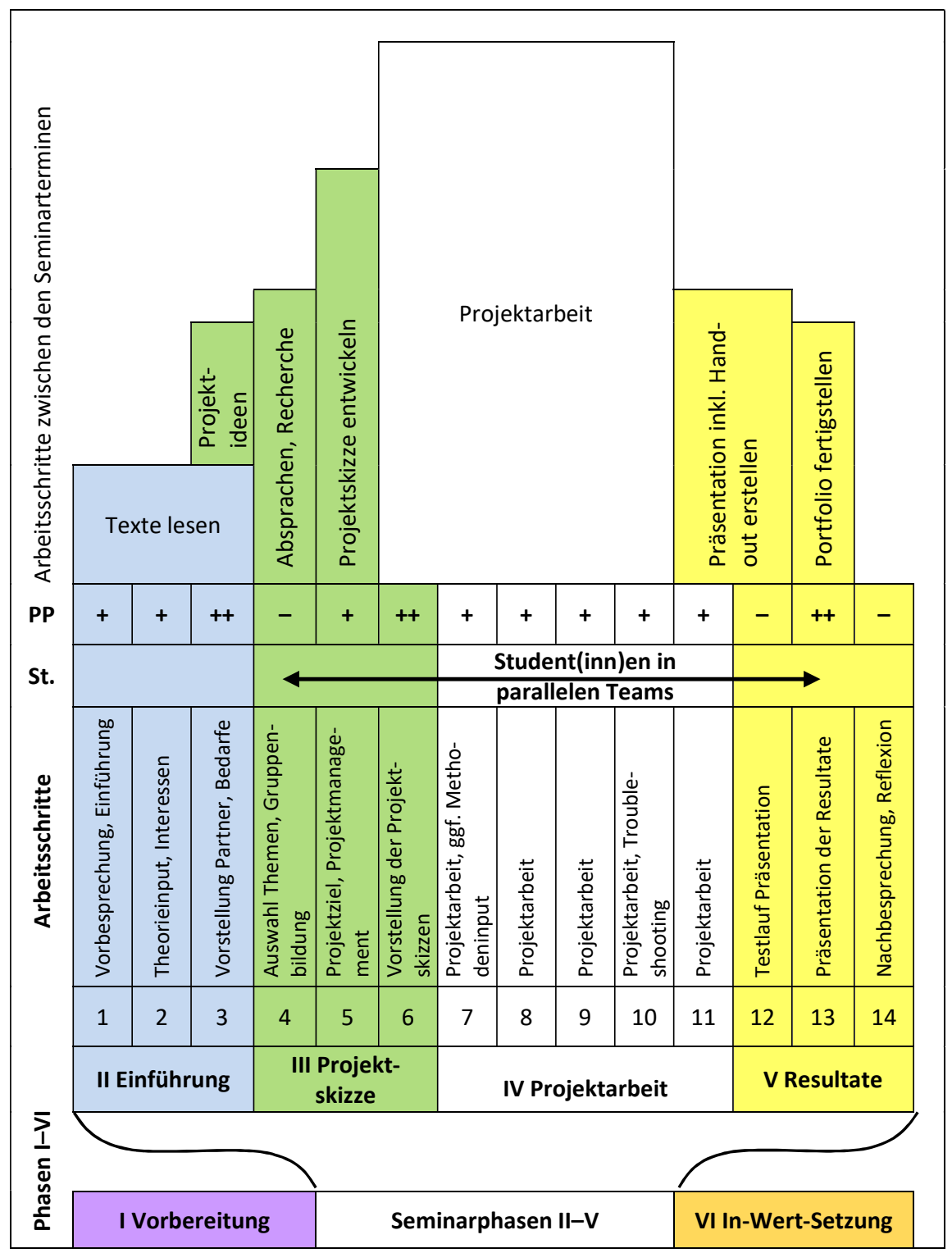

Abbildung 2: Phasen und Arbeitsschritte im Transformativen Projektseminar (idealtypisch, 14 Termine mit Student(inn)en umfassend). Die Zeile „PP“ zeigt, in welchen Phasen und Schritten die Praxispartner involviert sind, die Zeile „St.“ bezieht sich auf die Student(inn)en. (O Richard Beecroft. 


\subsection{Phase I: Vorbereitung}

In dieser Phase gilt es, ausgehend von einer vorläufigen Idee für das Seminar, den Einstieg in die gemeinsame Arbeit für alle beteiligten Gruppen zu gestalten: Auf Seiten der wissenschaftlichen Partner muss entschieden werden, wer aus dem wissenschaftlichen Projektrahmen als Lehrteam die Veranstaltung übernimmt. Wenn das TraPS u. a. der Verstetigung einer Kooperation mit bestimmten Praxispartnern dient, kann diese Entscheidung auch von der $\mathrm{Zu}$ - oder Absage der infrage kommenden Praxispartner abhängen. Es hat sich bewährt, dass im Lehrteam zumindest eine Person Erfahrung in projektförmiger Lehre hat und zumindest eine Person tiefergehende Kenntnisse im Gegenstandsfeld mitbringt. Das Lehrteam sollte Zeit dafür vorsehen, sich breit inhaltlich einzuarbeiten (da man nie genau weiß, wie die Projekte der Student(inn)en ausgerichtet sein werden) und die Rollen und Verantwortlichkeiten zu klären: Wer ist für die Student(inn)en für welche Fragen Ansprechperson, wer hält den Kontakt zu den Praxispartnern, wer bereitet welche Inputs vor, wer arbeitet sich in welche Themen tiefer ein? Gegebenenfalls können auch weitere Partner aus der Wissenschaft als Expert(inn)en eingebunden werden, da diese Rolle in der Regel eingespielt und unproblematisch anschlussfähig ist, solange sie nicht zu aufwendig wird.

Des Weiteren gilt es, Praxispartner einzubinden. Zunächst werden der thematische Rahmen und die Ziele, die im TraPS bearbeitet werden sollen, mit dem zu beteiligenden Praxispartner vereinbart. Im Anschluss muss vonseiten des Praxispartners (bzw. der Praxispartner, falls mehrere mitwirken werden) festgelegt werden, welche 1 bis 3 Personen sich im TraPS einbringen. Es sollte in Phase I verbindlich vereinbart werden, an welchen Terminen die Anwesenheit erforderlich ist und welche Inputs, insbesondere zu Beginn des Seminars, erfolgen sollen; eventuell kann hierfür ein Lehrauftrag oder ein Vortragshonorar vereinbart werden. Der Aufwand für die Praxispartner muss sich auch an deren Kapazitäten anpassen. Nur an drei Terminen eines TraPS ist die Anwesenheit unerlässlich: Bei der Vorstellung der Praxispartner (in Phase II), bei der Vorstellung der Projektskizzen (in Phase III) und bei der Präsentation und Übergabe der Resultate (in Phase V). Weiterhin gilt es zu entscheiden, wann und über welche Kommunikationsmittel die Praxispartner für die Student(inn)en erreichbar sein werden. Es sollte vereinbart werden, wann weitere Mitglieder der Akteursgruppen zu einzelnen Terminen im TraPS hinzukommen - dies ist insbesondere bei der Präsentation der Ergebnisse sinnvoll. Im Rahmen dieser vorbereitenden Absprachen sollten auch die Erwartungen seitens der Praxispartner diskutiert und zu hohe oder falsche Erwartungen korrigiert werden (z. B., dass eine reine Beratungs- oder Dienstleistungstätigkeit in Aussicht steht). 
Mit Blick auf die Student(inn)en muss in dieser Phase das Informations- und gegebenenfalls Werbematerial zum TraPS erstellt werden. Dies sollte mit großer Sorgfalt geschehen, da die Erwartungen, die sich aus den Veranstaltungsankündigungen ergeben, für die Teilnahmeentscheidung und die Zufriedenheit mit der Veranstaltung wichtig sind. Sowohl das Thema als auch die spezifische Arbeitsweise sollten deutlich herausgestellt werden. Daneben müssen natürlich alle normalen Vorbereitungen für eine Lehrveranstaltung erfolgen (z. B. Teilnahmelisten erstellen, Texte auf eine Lernplattform hochladen). Hierbei gilt es immer, darauf $\mathrm{zu}$ achten, dass die Praxispartner nicht außen vor bleiben bei der Nutzung universitätsinterner Infrastrukturen (z. B. Zugriffsrechte auf die Lernplattform). Ein rechtzeitiges und verbindliches Anmeldesystem erleichtert die Planung deutlich.

Sofern die Möglichkeit hierzu besteht, sollte in dieser Phase auch die Lernumgebung vorbereitet werden (s. Abschnitt 4.3), indem überlegt und entschieden wird, in welchen physischen Räumen, gegebenenfalls auch über welche virtuellen Plattformen, die Kooperation gestaltet werden soll und welche Materialien von Anfang an verfügbar sein sollten. Typische Fragen dieser Art in den Karlsruher TraPS betrafen etwa:

- Sollen die Student(inn)en die frei verfügbare Projektmanagementsoftware der Praxispartner nutzen, um den Anschluss an die Praxis zu erleichtern?

- Wo erhält man einen Stadtplan in DIN A0?

- Trifft man sich zu ausgewählten Terminen in den Räumen der Praxispartner?

- Mit welcher Cloud-Lösung dürfen Mitarbeiter(innen) der Kommune arbeiten?

- Wo lagern wir die erarbeiteten Materialien in einem vielfältig genutzten Raum?

Der Vorlauf und der Zeitaufwand für Phase I ist äußerst kontextabhängig. Eine vorausgegangene oder kontinuierlich bestehende Kooperation mit einem Praxispartner kann Phase I eines TraPS deutlich erleichtern. Wenn das TraPS verpflichtend in der Lehre verankert ist und dementsprechend nicht ausfallen darf, muss die Planung dafür weit im Voraus erfolgen - eventuell kann nicht jeder Praxispartner so weit im Voraus verbindliche Zusagen treffen.

\subsection{Phase II: Einführung}

Phase II beginnt mit der ersten regulären Seminarsitzung (90 Minuten, Uhrzeit in Abstimmung mit Praxispartnern) und umfasst drei Wochen (Termine 1 bis 3). Nachstehend werden die inhaltlichen Schwerpunkte der drei Termine geschildert. Dabei gilt jedoch, dass diese relativ einfach getauscht werden können, um beispielsweise eine Veranstaltung der Praxispartner besuchen zu können oder um 
,trockenen' theoretischen Input auf mehrere Termine zu verteilen. Die Praxispartner müssen unbedingt zu ihrer Vorstellung erscheinen, zu den anderen Terminen ist die Anwesenheit erwünscht, aber nicht erforderlich.

\section{Termin 1}

Die Student(inn)en erhalten eine Einführung in den übergreifenden thematischen Horizont und den wissenschaftlichen Rahmen (in den Karlsruher TraPS z. B.: Nachhaltigkeit und die Bedeutung von Städten, Bezug zum jeweiligen Themenfeld, z. B. nachhaltiger Konsum, sowie eine anschauliche Vorstellung des Reallabors). Hierzu werden auch Texte zur Verfügung gestellt, die von den Student(inn)en zwischen den Terminen gelesen werden sollen. Die Student(inn)en werden eingeführt in die transdisziplinäre Methodik des TraPS. Die zu erbringenden Studienleistungen - Projektergebnis, Lernportfolio und gegebenenfalls Hausarbeit (s. Abschnitt 4.1) - müssen erläutert werden. Hierzu typische formale Fragen zu Fristen, Bedingungen für Studienleistungen etc. müssen mit den Student(inn)en geklärt werden. Insbesondere sollte der hohe Arbeitsaufwand in Phase IV von Anfang an deutlich gemacht werden. Sofern der Kurs nicht verpflich-

tend ist, sollte seitens der Student(inn)en bis zum Ende von Phase II eine definitive Entscheidung über die Teilnahme erfolgen.

\section{Termin 2}

Am zweiten Termin werden theoretische Grundlagen knapp eingeführt, die für die Bearbeitung der Themenstellung unerlässlich sind. Bei Bedarf können auch grundlegende Wissensbestände dargestellt werden, die die Relevanz des Projekts deutlich machen (z. B. Informationen über Lebensmittelverschwendung zur Kooperation mit einer Tafel, Diskussion der Kritik an geplanter Obsoleszenz als Grundlage zur Kooperation mit einem Reparaturcafé).

Ein Element aus dem forschenden Lernen (s. Abschnitt 3.2), das sich in den TraPS besonders bewährt hat im Anschluss an theoretischen Input, ist das Explizieren und Reflektieren von eigenen Bildungszielen und Interessen durch die Student(inn)en. Dies wird durch eine kurze Phase individueller Reflexion, eventuell gefolgt von kurzen Zweiergesprächen zum Aktivieren und dann einem Austausch im Plenum erreicht. Hierbei kann die oben eingeführte Unterscheidung in Bildungs-, Praxis- und Forschungsziele als Reflexionsfolie verwendet werden, indem die Student(inn)en dazu aufgefordert werden, sich mit den eigenen Interessen in diesen Zieldimensionen zu positionieren (z. B. visualisiert mit Klebepunkten und Schlagworten in einem ,Zieldreieck' auf einem Flipchart). Die Student(inn)en halten ihre Ziele und Erwartungen individuell schriftlich fest und legen sie im Lernportfolio ab (s. Abschnitt 4.1), um sie in der Abschlussreflexion (Phase V) nutzen zu können. Wenn Praxispartner bei diesem Schritt anwesend 
sind, sollten sie sich weitgehend zurückhalten. Für das Lehrteam ist eine sokratische Gesprächsführung (s. Abschnitt 4.2) zielführend, eventuell verbunden mit einer Rollenverteilung unter den Lehrenden (z. B. Protokollieren und Moderieren oder Fokus auf die unterschiedlichen Zieldimensionen).

\section{Termin 3}

Die Praxispartner stellen ihre Arbeit vor, im Idealfall sowohl durch einen Vortrag als auch im Rahmen einer Exkursion, und beschreiben ihre Bedarfe. Gegebenenfalls können hierzu unterschiedliche Quellen genutzt werden, nicht nur klassische wissenschaftliche Texte (z. B. im TraPS zu „Gemeinwohlökonomie in Karlsruhe?" ein Video mit dem Gründer der GWÖ und eine Broschüre mit einer radikalen Kritik des Ansatzes durch eine wirtschaftsliberale Stiftung). Es kann passieren, dass sich relevante Probleme als nicht bearbeitbar für die teilnehmenden Student(inn)en herausstellen: In der Zusammenarbeit mit einer der Karlsruher Tafeln wurde etwa das Problem angesprochen, dass viele ältere Kunden nur wenige Einkäufe mit der Straßenbahn transportieren können, da deren Stufen zu hoch sind für Einkaufsroller. Da die Tafel bereits sehr gute und vertrauensvolle Kontakte zur Stadt hat und aus vielen Gründen in ihren gegenwärtigen Räumen bleiben muss, war durch die Student(inn)en kein Ansatzpunkt für einen barrierefreien Zugang zur Tafel zu identifizieren.

\subsection{Phase III: Projektskizze}

Ziel von Phase III ist es, dass die Student(inn)en Projektideen entwickeln und Studierendenteams bilden, um jeweils eine Projektidee zu einer Projektskizze auszuarbeiten, die realisierbar, relevant und interessant ist (und in diesem Sinne Potenzial bietet für Praxis-, Forschungs- und Bildungsziele). Phase III umfasst drei Seminartermine (Termine 4 bis 6). Direkt im Anschluss an den letzten Seminartermin von Phase II erhalten die Student(inn)en den Auftrag, individuell mehrere Projektideen zu formulieren, und zwar unabhängig davon, ob sie diese selbst bearbeiten wollen. Auch unvollständige Projektideen sind hier willkommen, die beispielsweise nur das Thema, aber kein Vorgehen beschreiben.

\section{Termin 4}

Der vierte Termin ist für den Projektverlauf besonders wichtig. Soweit irgend möglich, sollten alle Student(inn)en anwesend und notfalls bereit sein, etwas zu überziehen. Je nach Anzahl und Qualität der individuell gesammelten Projektideen ist noch ein gemeinsames Brainstorming nötig, zumindest sollte kurz Gelegenheit gegeben werden, neu aufkommende Ideen einzubringen. Alle Projektideen werden auf Moderationskarten festgehalten und thematisch gruppiert. 


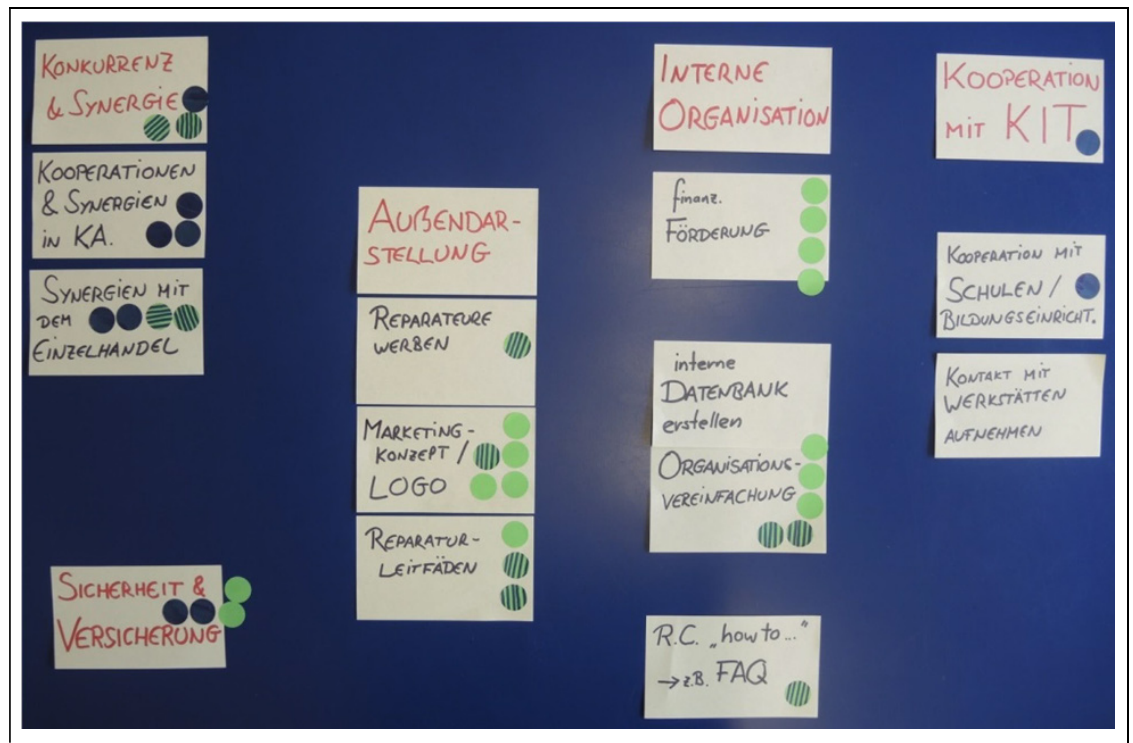

Abbildung 3: Geclusterte Projektideen, bewertet nach Bildungspotenzial (gestreift), Forschungspotenzial (dunkelblau) und Praxispotenzial (hellgrün). (C) Richard Beecroft.

Gegebenenfalls kann anhand dieser übersichtlicheren Sammlung noch einmal nach Themen gefragt werden, die noch fehlen, insbesondere auch im Hinblick auf die eingeführte Theorie und den Bedarf der Praxispartner.

Die so erarbeitete Ideensammlung wird nun durch die Student(inn)en nach drei Aspekten bewertet: Nach der (individuellen) Relevanz für den je eigenen Bildungsprozess, nach dem angenommenen praktischen Potenzial (in der Regel für den Praxispartner) und nach der wissenschaftlichen Relevanz der Themen (gegebenenfalls erfolgt diese Bewertung durch das Lehrteam, falls die Student(inn)en wenig einschlägige wissenschaftliche Vorkenntnisse mitbringen). Abb. 3 zeigt ein Beispiel für diesen Auswahlprozess. Auffälligkeiten in Bezug auf die Verteilung der Punkte werden gemeinsam diskutiert. Erst ausgehend von dieser Themenkarte finden sich dann Studierendenteams zusammen, die eine (oder gegebenenfalls auch mehrere zusammenpassende) dieser Ideen bearbeiten möchten. Wenn möglich sollten die Studierendenteams sich auf mehrere Cluster verteilen, zwingend ist aber nur, dass die Projekte hinreichend unterschiedlich sind - das kann auch gegeben sein, wenn mehrere Teams dasselbe Thema bearbeiten (z. B. erstellte ein Studierendenteam für das ReparaturCafé Karlsruhe, das vor einer Vereinsgründung stand, eine Best-Practice-Studie für Reparaturcafés in Deutschland, ein weiteres Studierendenteam ein Planspiel zum Test, ob die für das ReparaturCafé 
geplante Vereinssatzung die relevanten Fragen und Probleme behandelt; beide Projekte bezogen sich auf die Projektidee „Entwicklung der Satzung unterstützen"). In der Weiterentwicklung der Projektidee zur Projektskizze sollten die Student(inn)en Forschungs-, Bildungs- und Praxisziele berücksichtigen, auch wenn hierzu zunächst keine Punkte vergeben wurden.

Die Studierendenteams sollten eher klein sein (4 bis 5 Personen), um die Logistik für Treffen in der Projektphase nicht zu erschweren. Die Priorisierung der Themen- vor der Gruppenfindung erlaubt es, Interessen und Kompetenzen bestmöglich zu verteilen. Zudem besteht bei einem freiwilligen Kursangebot immer eine gewisse Gefahr, dass Student(inn)en doch auf die weitere Teilnahme verzichten oder erst spät zum Seminar dazustoßen, so dass eine zu frühe Gruppenzuordnung riskant ist. Durch die relativ späte Teambildung erhalten die Student(inn)en genug Zeit, sich kennenzulernen und die Seminargruppe als Ganzes zu erleben, nicht als konkurrierende Teams.

Es hat sich bewährt, den sich findenden Gruppen etwas Zeit zu geben, um miteinander abzustimmen, wer das Seminar für sich wie hoch priorisiert und wie viel Zeit und Energie bei den Einzelnen für die Projektarbeit zur Verfügung stehen hierzu sollten das Lehrteam und die Praxispartner möglichst den Raum verlassen, damit die Student(inn)en offen auch über eine eher geringe Beteiligung sprechen können. Insbesondere bei einer Gruppe, in der einige Student(inn)en keine Bescheinigung brauchen, andere die Veranstaltung aber als Prüfungsleistung in ihr Studium einbringen wollen, können sich Spannungen ergeben, die sich zu Beginn der Gruppenzusammensetzung am besten adressieren lassen.

$\mathrm{Ab}$ diesem Termin arbeiten die Student(inn)en als Teams zusammen. Damit ist die Akteurskonstellation im Seminar fertig aufgebaut (s. Abb. 1). Die Studierendenteams sollten sofort festlegen, durch welche Medien und in welcher Form (Treffen, Datenaustausch, Videokonferenzen etc.) sie zusammenarbeiten wollen; für Aspekte der Datensicherheit lohnt sich eventuell die Nutzung geschützter Cloud-Dienste, übliche Lernplattformen sind dagegen oft zu unflexibel zur Unterstützung von Projektarbeit.

\section{Termin 5}

Ausgehend von den von ihnen gewählte(n) Idee(n) arbeitet jedes Studierendenteam eine realistische Projektskizze aus. Diese umfasst die verfolgten Ziele, das angestrebte konkrete Ergebnis, die wichtigsten Arbeitspakete, die zu erwartenden Hürden und die Verteilung von Verantwortlichkeiten. Hierbei lohnt es sich, einerseits mit jeder Gruppe einzeln ihre Skizze durchzugehen (das Lehrteam sollte sich hierzu aufteilen), andererseits im Plenum übergreifende Fragen und Berührungspunkte zwischen den Projektskizzen (Synergien nutzen, Doppelarbeit vermeiden) anzusprechen - prinzipiell sind auch direkt aufeinander bezogene Projekte von 
Studierendenteams möglich, solange sie nicht zeitlich voneinander abhängen, also parallel bearbeitet werden können. Die Projektskizzen sollten alle einer festgelegten Form genügen (z. B. Handout von 2 Seiten, das die oben genannten Punkte aufgreift und separat Fragen ausweist, die mit den Praxispartnern zu klären sind). Bei manchen Arbeitsweisen (z. B. Fragebogenerstellung, Projektplanung) ist gegebenenfalls eine intensivere Unterstützung der Student(inn)en nötig. Bei diesem Termin sollten die Praxispartner eher nicht dabei sein.

\section{Termin 6}

Die Projektskizzen aller Studierendenteams werden den Praxispartnern gemeinsam vorgestellt, jeweils anhand eines Handouts, vorzugsweise mit einer kurzen Präsentation (jeweils 3 bis 4 Folien), die derselben Struktur wie das Handout folgt. $\mathrm{Zu}$ diesem Termin ist es von Vorteil, wenn die Praxispartner weitere Mitglieder ihrer Akteursgruppe einladen und eventuell auch weitere Wissenschaftler(innen) anwesend sind. Die Projektskizzen werden einzeln vorgestellt (ca. 10 Min./Projekt) und mit allen Anwesenden diskutiert (ca. 10 Min./Projekt), wobei die Praxispartner ausreichend zu Wort kommen sollten. Die Diskussionsergebnisse sollte jedes Studierendenteam für sich festhalten. Die (gegebenenfalls modifizierten) Projekte sind ab diesem Moment festgeschrieben, weitere Veränderungen müssen mit dem Lehrteam abgesprochen werden (solche sind, als Folge unerwarteter Entwicklungen in den Projekten, eher die Regel als die Ausnahme).

\subsection{Phase IV: Projektarbeit}

Die Projektarbeit umfasst fünf Wochen (Termine 7 bis 11). Das ist die Phase, in der die Studierendenteams ihre Projekte realisieren. In dieser überaus intensiven Phase dominiert ein selbstorganisierter Arbeitsstil, die gemeinsamen Seminartermine dienen primär der Unterstützung der Studierendenteams durch das Lehrteam sowie der Bearbeitung übergreifender Fragen oder möglicher Synergien, dem Austausch zwischen den Studierendenteams und kurzen methodischen Inputs seitens des Lehrteams, die mehrere Studierendenteams betreffen.

\section{Termine 7-11}

An diesen Seminarterminen wird in der Regel in einer kurzen Runde zu Beginn jedes Termins der Stand der Projekte vorgestellt und gegebenenfalls bestehender Klärungsbedarf benannt. Die einzelnen Studierendenteams erhalten insbesondere am 7. Termin methodische Beratung, ansonsten nach Bedarf Rückmeldung zu Zwischenergebnissen, inhaltliche Inputs oder Gelegenheit zum Austausch mit den Praxispartnern. Danach teilen sich die Student(inn)en wieder in ihre Teams auf, an mehrere Tische oder in getrennte Räume. 
Im gedrängten Semester stellt das Zeitmanagement oft die größte Herausforderung in dieser Phase dar. Dies ist insbesondere heikel, wenn Informationen von Dritten nötig sind und diese spät oder nicht zur Verfügung gestellt werden. Wenn die Praxispartner in dieser Phase nicht anwesend sind, sollten sie zumindest gut erreichbar sein (das Lehrteam natürlich ebenso). Die Student(inn)en müssen in dieser Phase in engem Austausch miteinander stehen; wenn ein Studierendenteam aber angekündigt einen Seminartermin nicht wahrnimmt, um etwa die Zeit für einen gemeinsamen Termin mit Dritten zu nutzen, ist dies in der Regel unproblematisch. Fehlt dagegen ein(e) Teilnehmer(in), ohne mit dem eigenen Team gesprochen und gegebenenfalls benötigte Zwischenergebnisse übergeben zu haben, ist das ein Alarmzeichen. Gelegentlich sind Interventionen im Gruppenprozess einzelner Teams nötig, beispielsweise eine Aussprache über Unmut über den Projektverlauf oder die Teamdynamik. Dabei hat die Erfahrung in den TraPS in Karlsruhe aber kein wiederkehrendes Muster gezeigt, wann oder aus welchen Anlässen ein Tief im Gruppenprozess eines Teams entstand. Das Team Teaching (s. Abschnitt 3.3) kann während der Projektarbeit dazu genutzt werden, den einzelnen Studierendengruppen ausreichend Raum zum Austausch mit den Lehrenden zu bieten, indem diese den Studierendenteams einzeln zur Verfügung stehen (und sich dann zwischen den Seminarterminen abstimmen). In dieser Phase kann es auch immer wieder nötig sein, die Studierendenteams durch sokratische Gesprächsführung (s. Abschnitt 4.2) zu unterstützen, ohne ihnen dabei das Heft aus der Hand zu nehmen. Sofern Student(inn)en im Rahmen des Seminars Hausarbeiten anfertigen, besteht in der Projektphase Gelegenheit, mögliche Themen hierfür abzusprechen, um Synergien zwischen Projektarbeit und Hausarbeiten rechtzeitig einzuplanen.

\subsection{Phase V: Resultate}

Die Phase V dauert drei Wochen (Termine 12 bis 14). Direkt im Anschluss an Phase IV, zwischen dem 11. und 12. Termin, erstellen die Studierendenteams jeweils eine Präsentation zu ihrem Projektergebnis (ca. 10 Min., bei wenigen Studierendenteams im TraPS auch etwas länger). Die Präsentation wird begleitet von einem kurzen Handout, das die wichtigsten Aspekte des Resultats enthält. Dieses soll so gestaltet sein, dass die Praxispartner es nutzen und weitergeben können. Wenn das Resultat selbst die Form eines kurzen Textes hat, ist kein zusätzliches Handout notwendig. Manche Resultate haben die Form von Prozessen oder Aktivitäten (etwa: eine Schulungsveranstaltung in Phase VI) und erfordern klare Absprachen zwischen den Praxispartnern und der Studierendengruppe. Die Präsentation wird erst nach der Projektarbeit (Phase IV) erstellt, damit nicht die bei Student(inn)en gut eingespielte Arbeitsweise des Vorbereitens von Vorträgen Energie von der eigentlichen Projektarbeit abzieht. Das Lehrteam muss deutlich machen, dass die Präsentation nicht selbst das Ergebnis ist, sondern nur dazu dient, das Resultat der Projektarbeit vorzustellen. 


\section{Termin 12}

Beim Testlauf der Präsentation sollte seitens des Lehrteams insbesondere darauf geachtet werden, dass die für die Praxispartner relevanten Informationen klar dargestellt sind und dass die Beschreibung, wie die Studierendenteams zu ihrem jeweiligen Resultat gekommen sind, nicht zu viel Raum einnimmt. An diesem Termin sollten die Student(inn)en auch angeregt werden, sich gegenseitig Feedback zu geben. Die Situation vor der echten Präsentation ist besonders günstig, konstruktives Feedback zu geben, da es direkt umgesetzt werden kann. Je nach Nähe der Projekte zueinander muss abgesprochen werden, welches Studierendenteam überlappende Inhalte präsentiert. Pragmatische Fragen zur Präsentation („Wer druckt die Handouts?“, „Welche Anschlüsse hat der Beamer?") sollten an diesem Termin final geklärt werden. Die Praxispartner sind an diesem Termin möglichst nicht anwesend.

\section{Termin 13}

Die Resultate werden den Praxispartnern präsentiert, weitere Personen können aber gut einbezogen werden. Im Idealfall sind die Praxispartner zu diesem Termin mit weiteren Personen vertreten, um eine praktische Nutzung der Resultate zu fördern. Der Ort und die Zeit für die Abschlusspräsentation müssen sorgfältig auf den Bedarf der Praxispartner abgestimmt werden: Soll es ein Abendtermin sein oder soll der Termin während der Arbeitszeit stattfinden? Steht ohnehin ein Treffen an, das sich mit dem Seminar verbinden lässt, oder sollte man konkurrierenden Terminen ausweichen?

Sofern ein materielles Produkt erarbeitet wurde, wird dieses übergeben. An jede Präsentation schließt sich eine zweigeteilte Diskussion an: Zunächst steht die Anwendung der Ergebnisse durch die Praxispartner im Vordergrund. Dabei reicht die Spanne der möglicherweise zu diskutierenden Themen von kleinen praktischen Fragen (z. B. „Wer lädt den Film auf welche Plattform hoch?“) über Fragen zum Anwendungsprozedere (z. B. „Wenn wir Ihr Planspiel nutzen, kann jemand von Ihnen moderieren?") bis hin zu grundlegenden Fragen zum Projektansatz. Wenn Projekte nicht vollends fertig geworden sind, sollte an diesem Punkt der Weg bis zum Abschluss geklärt werden. In einem zweiten Teil der Diskussion geben die Praxispartner den Studierendenteams Rückmeldung zur praktischen Relevanz ihrer Projektergebnisse.

Für die Student(inn)en stellt die Ergebnispräsentation den Kulminationspunkt ihres Engagements im Seminar dar. Alles, was danach noch stattfindet, sollte gut begründet werden, eine entspannte Atmosphäre bieten und wieder ganz unter der Regie des Lehrteams stattfinden. Unbedingt sollte eine Nachbesprechung mit den Student(inn)en stattfinden, um eine Reihe von Punkten zu bearbeiten (s. Termin 14). Dies sollte schon zu Beginn des TraPS angekündigt werden, um deutlich zu 
machen, dass es nicht nur um einen symbolischen Abschluss geht (in den Worten eines Studenten in einem Karlsruher TraPS: „Labern wir nur oder passiert da noch was?"). Wenn zwischen Präsentation der Resultate und Reflexion zwei bis drei Wochen (ohne Seminartermine) liegen, ist das eher vorteilhaft.

\section{Termin 14}

An diesem letzten regulären Termin sollten folgende Punkte jeweils knapp bearbeitet werden:

- Reflexion der Zusammenarbeit mit den Praxispartnern: Wie wurde die Kooperation erlebt, wie standen die Erfahrungen in Relation zu den Erwartungen, gab es unausgesprochene Konflikte? Grundlage hierzu sind die eingangs seitens der Student(inn)en individuell formulierten Ziele und Erwartungen (Phase II).

- Reflexion des Bildungsprozesses: Anhand des eigenen Lernportfolios (s. Abschnitt 4.1) können Student(inn)en den eigenen Lernprozess reflektieren. Ein methodischer Weg, um in diesem Arbeitsschritt auf das Individuum zu fokussieren, ist, die Student(inn)en vor einem Austausch im Plenum drei Reflexionsfragen in wenigen Sätzen schriftlich (fürs Lernportfolio) beantworten zu lassen. Diese Fragen sollten sich möglichst konkret auf den Prozess beziehen und Aspekte aufgreifen, die dem Lehrteam wesentlich erscheinen (eher „Inwieweit war die kritische Rückmeldung der Praxispartner auf Ihre Projektskizze für Sie hilfreich?“ als „Welche Rolle spielte Kritik in Ihrem Prozess?“). Die damit finalisierten Lernportfolios werden an diesem Termin abgegeben.

- Rückmeldung zum Seminarverlauf und Einordnung in den Forschungskontext: Die Student(inn)en erwarten in der Regel, dass sie zumindest in groben Zügen erfahren, wie ihre Projekte im Forschungskontext aufgegriffen werden, wie ihre Aktivitäten in einem weiteren Forschungshorizont einzuordnen sind und was das Lehrteam im Prozess gelernt hat. Diese Rückmeldung kann, muss aber nicht eine Bewertung der einzelnen Teams umfassen. Wenn es in oder zwischen den Studierendenteams Spannungen gab, kann hierzu auch eine Aussprache darüber im Plenum und/oder eine Reihe von getrennten Gesprächen mit den Studierendenteams erfolgen. In einigen Fällen fordern die Student(inn)en auch eine Gesamtbeurteilung der Lehrenden ein („Wie waren wir denn?"). Diese Frage lässt sich recht gut entlang der Phasen beantworten.

- Abschließend ist auch eine klassische Lehrevaluation der Lehrveranstaltung durch die Student(inn)en sinnvoll - diese ist in den meisten Universitäten und Hochschulen hoch formalisiert, so dass hier nicht im Detail darauf eingegangen wird. Sie kann durch eine kurze Abschlussrunde ergänzt werden, die persönliche Rückmeldung an die Lehrenden gibt und/oder strukturierten Leitfragen folgt. 
- Sofern Student(inn)en im Rahmen des Seminars Hausarbeiten anfertigen, sollten am Reflexionstermin hierzu Absprachen getroffen werden: Was soll bis wann bearbeitet werden? Dient die Hausarbeit primär Forschungszielen oder sollen auch Bildungs- und Praxisziele verfolgt werden? Soll die Hausarbeit nur an das Lehrteam gehen oder ist sie auch für die Praxispartner von Interesse?

\subsection{Phase VI: In-Wert-Setzung}

Diese Phase gerät leicht aus dem Blick, es ist aber äußerst riskant, hierfür keine zeitlichen Ressourcen einzuplanen. Sie dient dazu, die im TraPS verwobenen Stränge von Praxis, Forschung und Bildung wieder so voneinander zu lösen, dass die Bilanz für alle Zieldimensionen positiv ausfällt, zumindest aber in keiner Schaden entsteht.

Es gilt zunächst, die Fertigstellung oder Optimierung derjenigen Ergebnisse und Produkte zu begleiten, die noch nicht vollständig praxistauglich sind (ein typischer Fall: Ein Informationsflyer wird erstellt, aber die Klärung der Bildrechte ist nicht abgeschlossen). Teilweise müssen dabei auch die Eigentumsverhältnisse und die zukünftige Trägerschaft ausgehandelt werden. Wer hat zum Beispiel welche Rechte an einem im Seminar produzierten Film: die Student(inn)en, die ihn erstellten, die Praxispartner, für die er erstellt wurde, oder das Lehrteam, an das die externen Anfragen zur Nutzung des Films gestellt werden? In manchen Fällen ist es nötig, dass hierbei noch Student(inn)en - zumindest per E-Mail - involviert bleiben.

Es liegt in aller Regel in der Hand der Praxispartner, die erarbeiteten Ergebnisse zu nutzen. Dies kann sehr einfach (z. B. Aufhängen eines Infoposters) oder sehr aufwendig sein (z. B. Kooperation mit durch ein Studierendenteam rekrutierten Schulpartnern). Zum Abschluss sollte eine Nachbesprechung des Lehrteams mit den Praxispartnern durchgeführt werden, um die praktische Relevanz der Ergebnisse zu beurteilen, gegebenenfalls weitere Schritte in der Zusammenarbeit zu planen und sich - soweit gewünscht - wechselseitig Feedback über den Prozess zu geben.

Die normalen Lehraufgaben müssen abgeschlossen werden, wie das Betreuen der Hausarbeiten, das Benoten von Studienleistungen oder die Durchführung mündlicher Prüfungen.

Die erarbeiteten Ergebnisse gilt es auch in den Forschungsprozess einzuspeisen. $\mathrm{Zu}$ den Arbeiten, die hierzu nötig sein können, gehören die Datenintegration (wie 
z. B. das Eintragen von Punkten in einer Online-Karte), die Entwicklung von Nutzungsstrategien für ausgearbeitete partizipative Methoden oder das Aufgreifen bislang unberücksichtigter Theoriekonzepte.

Einzelne Student(inn)en suchen schließlich nach dem Seminar auch nach Gelegenheiten, weiter mit dem Lehrteam bzw. dessen Institution (bei den Karlsruher TraPS: dem Reallabor Karlsruhe) in Kontakt zu bleiben, beispielsweise über studentische oder zivilgesellschaftliche Initiativen - so kann das Seminar auch zur Entwicklung des Netzwerks von Akteuren beitragen.

Der Verlauf des TraPS muss immer wieder angepasst werden: Feiertage, Termine der Praxispartner, Exkursionswochen oder unabgeschlossene Arbeitsschritte machen immer wieder Anpassungen gegenüber dem idealtypischen Verlauf nötig, ebenso Sprünge zurück in frühere Phasen, wenn einzelne Punkte nicht abgeschlossen wurden. Es hat sich aber bewährt, diesen idealtypischen Ablaufplan im Seminar trotzdem sichtbar zu halten, nicht zuletzt, um den hohen Arbeitsaufwand während der Projektphase deutlich zu machen und gegebenenfalls bei den Student(inn)en einfordern zu können.

\section{Didaktische Ansätze als Grundlage im TraPS}

Das TraPS integriert vier Ansätze aus der allgemeinen Didaktik und Hochschuldidaktik (die in sich noch keine Lehrkonzeptionen darstellen), um transdisziplinäres Arbeiten mit transformativem Anspruch zu ermöglichen:

- Service Learning beschreibt Lehre, die den Student(inn)en einen Lernprozess durch Erbringen gesellschaftlich relevanter Leistungen eröffnet (s. Abschnitt $3.1)$.

- Im Forschenden Lernen verfolgen die Student(inn)en eigene Interessen und Ideen in einer wissenschaftlichen Arbeitsweise (s. Abschnitt 3.2).

- Im Team Teaching gestalten Lehrende mit unterschiedlichen Perspektiven, unterschiedlichem Wissen und unterschiedlichen Rollen gemeinsam einen Bildungsprozess (s. Abschnitt 3.3).

- Durch Soziales Lernen können die unterschiedlichen Beteiligten im Austausch von- und miteinander lernen (s. Abschnitt 3.4). 
Diese Ansätze werden in diesem Kapitel kurz vorgestellt, wobei zuerst ihre spezifische Funktion im TraPS anhand von Qualitätskriterien erläutert wird und anschließend das Zusammenspiel der didaktischen Ansätze dargestellt wird (s. Abschnitt 3.5). Im folgenden Kapitel werden drei didaktische Einzelthemen angesprochen, die sich im TraPS als wichtig herausgestellt haben (s. Kapitel 4):

- Die Vermeidung von negativen Rückwirkungen der Prüfungsform, u. a. mit Lernportfolios.

- Das Sokratische Gespräch als eine Art der Gesprächsführung, in der das Lehrteam durch Fragen die Student(inn)en unterstützt, ohne ihren Ergebnissen vorzugreifen.

- Die Lernumgebung so zu gestalten, dass sie die Prozesse im TraPS unterstützt.

\subsection{Service Learning}

Der Ansatz Service Learning wurde in den 2000er Jahren aus den USA nach Deutschland gebracht, unter anderem unter dem Begriff „Lernen durch Engagement", wobei er sich im Schul- und Hochschulkontext relativ unabhängig voneinander weiterentwickelte. Clayton Hurd definiert Service Learning wie folgt:

„As pedagogy, service learning emphasizes meaningful student learning through applied, active, project-based learning that draws on multiple knowledge sources (academic, student knowledge and experience, and community knowledge) and provides students with ample opportunities for ethical and critical reflection and practice." (Hurd 2008, S. 44).

Service Learning erreicht eine positive Wirkung im Lebensumfeld der Student(inn)en, indem diese einüben, Verantwortung zu übernehmen. Dabei ist das Wechselspiel von Erfahrung und Reflexion zentral. Im deutschsprachigen Diskurs finden die Qualitätskriterien von Seifert et al. (2012) weitgehend Zustimmung. Tab. 1 zeigt die Kriterien und deren jeweilige Umsetzung im TraPS. 
Tabelle 1: Elemente von Service Learning (nach Seifert et al. 2012) im TraPS.

\begin{tabular}{|c|c|c|}
\hline $\begin{array}{l}\text { Qualitätskriterien für } \\
\text { Service Learning }\end{array}$ & Umsetzung im TraPS & Phase \\
\hline $\begin{array}{l}\text { Das Engagement der } \\
\text { Lernenden reagiert auf } \\
\text { einen realen Bedarf in } \\
\text { der Gemeinschaft. }\end{array}$ & $\begin{array}{l}\text { - Praxispartner mit bearbeitbaren Bedarfen } \\
\text { - Bedarfe aus der Praxis werden vorgestellt } \\
\text { und diskutiert }\end{array}$ & I \\
\hline $\begin{array}{l}\text { Service Learning ist mit } \\
\text { den Inhalten der Lehr- } \\
\text { pläne verknüpft und } \\
\text { greift diese explizit auf. }\end{array}$ & $\begin{array}{l}\text { - Möglichst Einbettung in einen Studiengang, } \\
\text { ein fachübergreifendes Zertifikatsprogramm } \\
\text { o. Ä. }\end{array}$ & 1 \\
\hline $\begin{array}{l}\text { Es findet eine regelmäßi- } \\
\text { ge und bewusste Reflexi- } \\
\text { on der Erfahrungen statt. }\end{array}$ & $\begin{array}{l}\text { - Reflexion der individuellen Ziele } \\
\text { - Reflexion als Teil des Prozesses } \\
\text { - Abschlussreflexion ohne Praxispartner }\end{array}$ & $\begin{array}{l}\text { II } \\
\text { III, IV } \\
\text { V }\end{array}$ \\
\hline $\begin{array}{l}\text { Die Lernenden sind aktiv } \\
\text { in die Konzeption, Um- } \\
\text { setzung und Nachberei- } \\
\text { tung des Service Learn- } \\
\text { ing-Vorhabens eingebun- } \\
\text { den. }\end{array}$ & $\begin{array}{l}\text { - Abstimmung der Projektideen mit den Pra- } \\
\text { xispartnern } \\
\text { - } \text { Eigenständige Umsetzung durch die Stu- } \\
\text { dent(inn)en } \\
- \text { Diskussion der Umsetzbarkeit/Umsetzung } \\
\text { der Ergebnisse mit Praxispartnern } \\
- \text { Nachbereitung: Portfolios und Hausarbeiten }\end{array}$ & $\begin{array}{l}\text { III } \\
\text { VI, V } \\
\text { II, III, } \\
\text { VI }\end{array}$ \\
\hline $\begin{array}{l}\text { Das Engagement der Ler- } \\
\text { nenden findet gemein- } \\
\text { sam mit Partnern außer- } \\
\text { halb des (Hoch-)Schul- } \\
\text { kontextes statt. }\end{array}$ & $\begin{array}{l}\text { - Einbindung von Praxispartnern } \\
\text { - Gegebenenfalls Third Space zwischen Univer- } \\
\text { sität und Gesellschaft nutzen, z. B. Reallabor }\end{array}$ & $\begin{array}{l}\mathrm{I} \\
\mathrm{I}-\mathrm{VI}\end{array}$ \\
\hline $\begin{array}{l}\text { Die Lernenden erhalten } \\
\text { im Prozess und am Ende } \\
\text { des Service Learning- } \\
\text { Projekts konstruktives } \\
\text { Feedback und Anerken- } \\
\text { nung. }\end{array}$ & $\begin{array}{l}\text { - Feedback zu den Projektskizzen } \\
\text { - Begleitendes Feedback f. Studierendenteams } \\
\text { - Wechselseitiges Feedback: Testlauf Präsenta- } \\
\text { tion } \\
\text { - Feedback zu den Resultaten durch das Lehr- } \\
\text { team und die Praxispartner } \\
\text { - Feedback in der Abschlussreflexion }\end{array}$ & $\begin{array}{l}\text { III } \\
\text { IV } \\
\text { V } \\
\text { V }\end{array}$ \\
\hline
\end{tabular}




\subsection{Forschendes Lernen}

Unter dem Begriff Forschendes Lernen (learning through inquiry) werden zwei Ansätze - teils übereinstimmend, teils divergierend - diskutiert: zum einen die selbstbestimmte und aktive Einführung von Lernenden in das wissenschaftliche Arbeiten, zum anderen die didaktische Nutzung der strukturellen Parallelen des erfahrungsbasierten Lernens mit der Forschung (Mieg und Lehmann 2017). Beides ist für das TraPS interessant. Eine ausdifferenzierte Typologie forschungsbezogener Lehre bieten Rueß et al. (2016), einen Vorschlag zur Abgrenzung des forschenden Lernens vom forschungsbasierten oder forschungsorientierten Lernen macht Huber (2014). Folgende Definition beschreibt den Kern forschenden Lernens:

„Forschendes Lernen zeichnet sich vor anderen Lernformen dadurch aus, dass die Lernenden den Prozess eines Forschungsvorhabens, das auf die Gewinnung von auch für Dritte interessanten Erkenntnissen gerichtet ist, in seinen wesentlichen Phasen - von der Entwicklung der Fragen und Hypothesen über die Wahl und Ausführung der Methoden bis zur Prüfung und Darstellung der Ergebnisse in selbstständiger Arbeit oder in aktiver Mitarbeit in einem übergreifenden Projekt - (mit)gestalten, erfahren und reflektieren.“ (Huber 2009, S. 11).

Wegen der Verankerung des Diskurses zum Forschenden Lernen auch in der Schulpädagogik kommt der professionellen Reflexion der Lehrendenrolle darin besondere Bedeutung zu. Reitinger (2013) formuliert Qualitätskriterien für Forschendes Lernen, die in Tab. 2 dargestellt und den didaktischen Umsetzungen im TraPS gegenübergestellt sind (s. auch Reitinger et al. 2016). Abweichend vom klassischen Forschenden Lernen tritt im TraPS an die Stelle der Hypothesenbildung ein lösungsorientierter Ansatz, wie es Vilsmaier und Meyer (2017) für Forschendes Lernen in der Nachhaltigkeitsforschung vorgeschlagen haben. 
Tabelle 2: Elemente Forschenden Lernens (nach Reitinger 2013 und Reitinger et al. 2016) im TraPS.

\begin{tabular}{|c|c|c|}
\hline $\begin{array}{l}\text { Qualitätskriterien für } \\
\text { Forschendes Lernen }\end{array}$ & Umsetzung im TraPS & Phase \\
\hline $\begin{array}{l}\text { Neugierde/ } \\
\text { Forschungs- } \\
\text { interesse }\end{array}$ & $\begin{array}{l}\text { - Explikation der individuellen Interessen und } \\
\text { Ideen für Projekte } \\
\text { - Freie Themenwahl in Hausarbeiten }\end{array}$ & VI \\
\hline $\begin{array}{l}\text { Lernwege finden und } \\
\text { mitbestimmen }\end{array}$ & $\begin{array}{l}\text { - Einbezug kritischer Perspektiven, um Dogmatik } \\
\text { zu vermeiden } \\
\text { - Wahl des Projektthemas } \\
\text { - Themenfindungsprozess integriert Ideensamm- } \\
\text { lung, Bewertung, Auswahl und Gruppenbildung } \\
\text { - Erstellung der Projektskizze } \\
\text { - Selbstständige Projektrealisierung }\end{array}$ & $\begin{array}{l}\text { II } \\
\text { III } \\
\text { III } \\
\text { III } \\
\text { III, IV }\end{array}$ \\
\hline $\begin{array}{l}\text { Vermuten auf der } \\
\text { Basis von Erfahrung }\end{array}$ & $\begin{array}{l}\text { - Im Kern kein (!) hypothesenprüfendes Vorge- } \\
\text { hen, stattdessen Suche nach realistischen Prob- } \\
\text { lemlösungen } \\
\text { - Einbezug von Erfahrungen der Praxispartner }\end{array}$ & $\begin{array}{l}\text { III-V } \\
\mathrm{I}-\mathrm{VI}\end{array}$ \\
\hline $\begin{array}{l}\text { Authentizität, Auto- } \\
\text { nomie und Explora- } \\
\text { tion }\end{array}$ & $\begin{array}{l}\text { - Freiheit in der Projektgestaltung } \\
\text { - Spanne von explorativen bis zu konstruktiven } \\
\text { Projekten }\end{array}$ & $\begin{array}{l}\text { III, IV } \\
\text { III-V }\end{array}$ \\
\hline $\begin{array}{l}\text { Reflektierte Kommu- } \\
\text { nikation durch das } \\
\text { kritische Gespräch }\end{array}$ & $\begin{array}{l}\text { - Nutzung Sokratischer Gesprächsführung (s. } \\
\text { Abschnitt 4.2) } \\
\text { - Wechselseitige Rückmeldung zwischen Studie- } \\
\text { rendenteams } \\
\text { - Formative Evaluationsrunden, Abschlussreflexi- } \\
\text { on }\end{array}$ & $\begin{array}{l}\text { II-V } \\
\text { IV, V } \\
\text { V }\end{array}$ \\
\hline $\begin{array}{l}\text { Anwenden und Mit- } \\
\text { teilen (conclusion- } \\
\text { based transfer) }\end{array}$ & $\begin{array}{l}\text { - Präsentation und Ergebnisübergabe an die } \\
\text { Praxispartner } \\
\text { - Beteiligung an der In-Wert-Setzung }\end{array}$ & $\mathrm{V}$ \\
\hline
\end{tabular}




\subsection{Inter- und transdisziplinäres Team Teaching}

Team Teaching ist ein Ansatz, der kooperative Lehre durch mehrere Lehrende beschreibt. Auf Basis einer Literaturanalyse führen Welch et al. (1999) vielfältige Definitionen von Team Teaching und verwandten Ansätzen folgendermaßen zusammen:

„We define team teaching as the simultaneous presence of two educators in a classroom setting who share responsibility in the development, implementation, and evaluation of direct service in the form of an instructional or behavioral intervention to a group of students with diverse needs." (Welch et al. 1999, S. 38)

Im Team Teaching war die Beziehung zwischen den Lehrenden ursprünglich oft hierarchisch angelegt (Michael 1963), heute aber wird sie in der Regel kooperativ gedeutet. Besondere Bedeutung kommt dem Team Teaching in der interdisziplinären Lehre zu (DeZure 2010), sowie im Einbezug von externen Partnern (Higgins und Litzenberg 2015).

Im Fall des TraPS gilt es zwei Ebenen der Zusammenarbeit im Team Teaching zu berücksichtigen, erstens die zwischen den Wissenschaftler(inne)n im Lehrteam und zweitens deren Kooperation mit Praxispartnern. Auf diese Weise werden sowohl eine interdisziplinäre Perspektivenvielfalt im Lehrteam als auch eine transdisziplinäre Erweiterung ermöglicht. Im interdisziplinären Team Teaching sollten nicht nur die disziplinären Kompetenzen der Lehrenden berücksichtigt werden, sondern auch deren weitere Kompetenzen (z. B. Moderationsfähigkeiten, Softwarekenntnisse, Erfahrungen mit ähnlichen Projekten). Im transdisziplinären Team Teaching gilt es darüber hinaus zu berücksichtigen, wie viele theoretische Beiträge die Praxispartner beisteuern können und wollen - die Erfahrungen in den Karlsruhe TraPS waren hier sehr unterschiedlich. Darüber hinaus erlaubt das Team Teaching auch die Weitergabe von Erfahrungen im Lehrteam sowie wechselseitige Beobachtung und Rückmeldung. Die folgenden Charakteristika eignen sich dazu, gutes Team Teaching zu beschreiben, und lassen sich der Umsetzung in einem TraPS gegenüberstellen (s. Tab. 3). 
Tabelle 3: Charakteristika von Team Teaching (nach Murata 2002, S. 73f., eigene Übersetzung) im TraPS.

\begin{tabular}{|c|c|c|}
\hline $\begin{array}{l}\text { Charakteristika von } \\
\text { Team Teaching }\end{array}$ & Umsetzung im TraPS & Phase \\
\hline $\begin{array}{l}\text { Gemeinsame Lehr- } \\
\text { philosophie (com- } \\
\text { mon philosophy) }\end{array}$ & $\begin{array}{l}\text { - Normativer Orientierungsrahmen, z. B. Nachhal- } \\
\text { tigkeit } \\
\text { - Frühe Abstimmung der Ziele im Lehrteam } \\
\text { - }\end{array}$ & $\begin{array}{l}1 \\
1\end{array}$ \\
\hline $\begin{array}{l}\text { Betonung (über- } \\
\text { greifender) Kon- } \\
\text { zepte (emphasis } \\
\text { on concepts) }\end{array}$ & $\begin{array}{l}\text { - Übergeordnetes Thema, z. B. nachhaltige Stadt- } \\
\text { entwicklung als integrativ wirkendes Konzept } \\
\text { - Einführung von Schlüsselbegriffen } \\
\text { - Suche nach gemeinsamen Schlüsselkonzepten mit } \\
\text { den Praxispartnern (was nicht immer gelingt) }\end{array}$ & $\begin{array}{l}\mathrm{I}-\mathrm{VI} \\
\mathrm{II}\end{array}$ \\
\hline $\begin{array}{l}\text { Unterschiede } \\
\text { als Stärken } \\
\text { (differences as } \\
\text { strengths) }\end{array}$ & $\begin{array}{l}\text { - Aufstellung eines Lehrteams mit komplementären } \\
\text { Kompetenzen } \\
\text { - Darstellung der jeweiligen Kompetenzen gegen- } \\
\text { über den Student(inn)en } \\
\text { - Aufteilung, um unterschiedliche Lern- und Arbeits- } \\
\text { stile der Student(inn)en besser betreuen zu können }\end{array}$ & II, IV \\
\hline $\begin{array}{l}\text { Gemeinsame Pla- } \\
\text { nung (team plan- } \\
\text { ning) }\end{array}$ & $\begin{array}{l}\text { - Vor- und Nachbereitung jedes Seminartermins } \\
\text { - Gemeinsame Planung federt Fehlzeiten von Mit- } \\
\text { gliedern des Lehrteams gut ab } \\
\text { - Möglichkeit wechselseitiger Rückmeldung }\end{array}$ & $\begin{array}{l}I I-V \\
I I-V\end{array}$ \\
\hline $\begin{array}{l}\text { Offenheit für Ver- } \\
\text { änderungen (open- } \\
\text { ness to change) }\end{array}$ & $\begin{array}{l}\text { - Vor- und Nachbereitung erlaubt kontinuierliche } \\
\text { Kurskorrekturen im Seminarverlauf. }\end{array}$ & $\mathrm{II}-\mathrm{V}$ \\
\hline
\end{tabular}

\subsection{Soziales Lernen}

Die Diskurse zum Sozialen Lernen (social learning, societal learning) sind vielfältig und heterogen (s. Beiträge in Wals 2007), zu weiten Teilen auch ohne pädagogisch-didaktische Perspektive. Je nach Lesart bedeutet Soziales Lernen, dass unterschiedliche Personen miteinander, voneinander, in gemeinsamer Aktivität lernen oder dass Institutionen, Organisationen, Netzwerke oder die Gesellschaft als Ganzes ,lernen'. Für das TraPS sind zwar all diese Lesarten potenziell einschlägig, aber vier davon lassen sich didaktischen und methodischen Elementen eines TraPS gegenüberstellen (s. Tab. 4). 
Tabelle 4: Elemente Sozialen Lernens im TraPS.

\begin{tabular}{|c|c|c|}
\hline Soziales Lernen & Umsetzung im TraPS & Phase \\
\hline inder & $\begin{array}{l}\text { - Unterschiedlicher Hintergrund im Lehrteam, unter- } \\
\text { schiedliche Praxispartner } \\
\text { - Wenn möglich: Kooperation Student(inn)en ver- } \\
\text { schiedener Studiengänge } \\
\text { - } \\
\text { - Kustausch der Studierendenteams untereinander } \\
\text { Kompetenzen unter den Teilnehmer(inne)n explizie- } \\
\text { ren } \\
\text { - Einbezug nicht-akademischer Kompetenzen aller Be- } \\
\text { teiligten }\end{array}$ & $\begin{array}{l}\text { II } \\
I-I I I \\
I I I-V \\
I I, I I I\end{array}$ \\
\hline $\begin{array}{l}\text { Ler } \\
\text { mit }\end{array}$ & 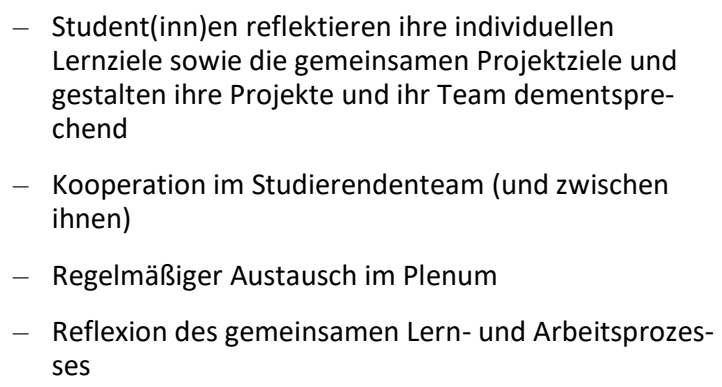 & $\begin{array}{l}I I I-\mathrm{V} \\
\mathrm{II-V} \\
\mathrm{III-V}\end{array}$ \\
\hline $\begin{array}{l}\text { Lernen in } \\
\text { gesellschaft- } \\
\text { lichen Zusam- } \\
\text { menhängen }\end{array}$ & $\begin{array}{l}\text { - Übergreifender Theorierahmen, z. B. nachhaltige } \\
\text { Entwicklung } \\
\text { - Reale gesellschaftliche Problemlagen als Referenz- } \\
\text { punkt } \\
\text { - Spezifische Bedarfe der Praxispartner } \\
\text { - Erleben der eigenen Wirksamkeit in praktischen } \\
\text { Kontexten }\end{array}$ & $\begin{array}{c}\text { I, V, VI } \\
\text { I, V, VI } \\
\text { I, II } \\
\text { III-VI }\end{array}$ \\
\hline $\begin{array}{l}\text { Alle Beteiligten } \\
\text { lernen im Trans- } \\
\text { formations- } \\
\text { prozess }\end{array}$ & $\begin{array}{l}\text { - Offenheit der Lehrenden, selbst zu lernen; Zuge- } \\
\text { ständnis eigener Wissenslücken und fehlender Kom- } \\
\text { petenzen } \\
\text { - Gelegenheit für die Praxispartner, aus der Seminarer- } \\
\text { fahrung zu lernen }\end{array}$ & $\mathrm{I}-\mathrm{VI}$ \\
\hline
\end{tabular}


Der erste Punkt, das Lernen voneinander, stellt in TraPS immer wieder eine besondere Herausforderung für die Planung dar, da nicht absehbar ist, mit welchen Fähigkeiten und welchem Wissen - und komplementär mit welchen Lernbedarfen - Student(inn)en in den Prozess eintreten werden.

\subsection{Integration der Ansätze}

Die oben vorgestellten Ansätze werden in einem TraPS verbunden. Die Vielfalt der didaktischen Elemente dient dazu, unterschiedliche Lernformen und Typen von Studierendenprojekten zu ermöglichen, aber auch als Sicherheitsnetz, denn sie bietet so viel Flexibilität, dass zumindest einige der Lehr- und Lernansätze sowie der angebotenen Herangehensweisen gelingen sollten und dass unterschiedliche Elemente den jeweiligen Interessen und Vorlieben der Student(inn)en entgegenkommen.

Abb. 4 verdeutlicht, wie die didaktischen Ansätze Verbindungen zwischen den drei Zieldimensionen des TraPS ermöglichen. Inter- und transdisziplinäres Team Teaching stellt eine Brücke zwischen Forschungs- und Praxiszielen dar, Forschendes Lernen greift eine an Forschungszielen orientierte methodische Arbeits-

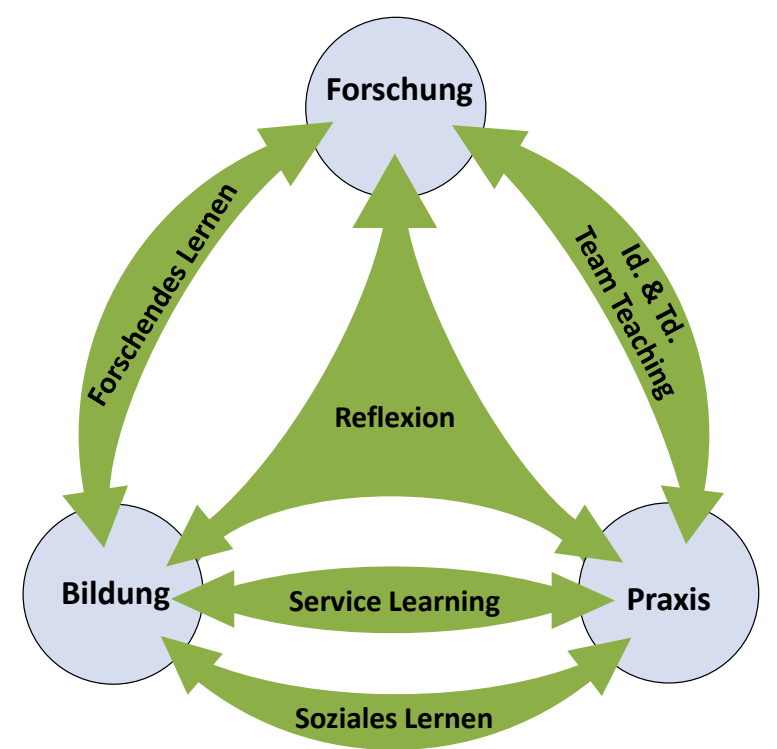

Abbildung 4: Die didaktischen Ansätze im TraPS verbinden die drei Zieldimensionen. (C) Richard Beecroft. 
weise auf und verbindet sie mit Bildungszielen, Service Learning wiederum richtet Bildungsprozesse an Praxiszielen aus. Soziales Lernen - in einer seiner Lesarten - nutzt die gemeinsame Praxis dafür, dass die Beteiligten von- und miteinander lernen können. In allen genannten didaktischen Ansätzen ist Reflexion der Erfahrungen essenziell. Sie stellt zugleich (durch Abstraktion) einen Mechanismus der Forschung und (z. B. durch kritisches Hinterfragen der eigenen Rolle) einen Mechanismus der Bildung dar.

Tab. 5 gibt eine Übersicht über die Ansätze und die jeweils im Detail übernommenen Kriterien und Anforderungen. Diese wurden hierfür sprachlich angeglichen und zu fünf Clustern zusammengefasst, die zentrale didaktische Felder im TraPS beschreiben. Für Lehrende, die nicht mit allen dieser Ansätze vertraut sind, dient die Tabelle dazu auszuloten, welche didaktische Vorbereitung noch nötig ist. Rechts zeigt die Tabelle, in welcher Phase das jeweilige Kriterium von besonderer Relevanz ist. Anhand der Tabelle wird deutlich, dass die Ansätze nicht einzelnen Phasen zugeordnet sind, sondern jeweils mehrere Phasen im Seminarablauf betreffen. Wenn Schwierigkeiten in einer der Phasen auftreten, bietet die Tabelle eine schnelle Zuordnung, auf welche Elemente der jeweiligen didaktischen Ansätze zurückgegriffen werden kann.

Um das Zusammenspiel der Ansätze zu realisieren, werden auch einige didaktische Methoden und Elemente eingesetzt, die vielleicht nicht allgemein bekannt sind. Sie sollen im Folgenden dargestellt werden. 
Tabelle 5: Übersicht über die im TraPS verbundenen didaktischen Ansätze (SeL: Service Learning, FL: Forschendes Lernen, TT: Team Teaching, SoL: Soziales Lernen).

\begin{tabular}{|c|c|c|c|c|c|c|c|c|c|c|c|}
\hline \multirow{2}{*}{\multicolumn{2}{|c|}{ Elemente }} & \multicolumn{4}{|c|}{$\begin{array}{c}\text { Didaktischer } \\
\text { Ansatz }\end{array}$} & \multicolumn{6}{|c|}{$\begin{array}{l}\text { TraPS } \\
\text { Phase }\end{array}$} \\
\hline & & Sel & FL & TT & Sol & 1 & II & III & IV & $\mathbf{v}$ & VI \\
\hline \multirow{3}{*}{$\begin{array}{l}\text { Dreifacher } \\
\text { Ausgangs- } \\
\text { punkt }\end{array}$} & Realer Bedarf & $x$ & & & $x$ & & & & & & \\
\hline & Wiss. Fragestellung & & $x$ & & & & & & & & \\
\hline & Neugierde & & $x$ & & $x$ & & & & & & \\
\hline \multirow{3}{*}{$\begin{array}{l}\text { Dreifache } \\
\text { Einbettung }\end{array}$} & Curriculare Einbettung & $x$ & $x$ & & & & & & & & \\
\hline & Einbettung in Forschung & & $x$ & & & & & & & & \\
\hline & Praktische Einbettung & $x$ & & & $x$ & & & & & & \\
\hline \multirow{3}{*}{$\begin{array}{l}\text { Aktive Rolle } \\
\text { der Lernen- } \\
\text { den }\end{array}$} & (Co-)Design & $x$ & $x$ & & & & & & & & \\
\hline & Durchführung & $x$ & $x$ & & $x$ & & & & & & \\
\hline & Anwenden/Weitergeben & $x$ & $x$ & & & & & & & & \\
\hline \multirow{9}{*}{$\begin{array}{l}\text { Vielfältige } \\
\text { Mechanismen } \\
\text { des Lernens }\end{array}$} & Instruktion/Input & & & $x$ & & & & & & & \\
\hline & Übergreifende Konzepte & & & $x$ & & & & & & & \\
\hline & Kritisches Gespräch & & $x$ & & & & & & & & \\
\hline & Reflexion & $x$ & $x$ & & $x$ & & & & & & \\
\hline & Hypothesen/Lösungsideen & & $x$ & & & & & & & & \\
\hline & Erfahrung & $x$ & $x$ & & & & & & & & \\
\hline & Wissenschaftl. Methoden & & $x$ & & & & & & & & \\
\hline & Feedback & $x$ & $x$ & & & & & & & & \\
\hline & Austausch/Differenz & $x$ & & $x$ & $x$ & & & & & & \\
\hline \multirow{5}{*}{$\begin{array}{l}\text { Gemeinsame } \\
\text { und reflexive } \\
\text { Aufgaben der } \\
\text { Lehrenden }\end{array}$} & Gemeins. Lehrphilosophie & & & $x$ & & & & & & & \\
\hline & Gemeinsame Planung & & & $x$ & & & & & & & \\
\hline & Offenheit f. Veränderung & & & $x$ & & & & & & & \\
\hline & Reflexion Lehrendenrolle & & $x$ & & & & & & & & \\
\hline & Evaluation & $x$ & & & & & & & & & \\
\hline
\end{tabular}




\section{Einzelne didaktische Methoden und Elemente im TraPS}

\subsection{Bewerten ohne negative Rückwirkungen}

Bewerten stellt ein zentrales - und nicht immer geliebtes - Thema jeder Lehre dar. Immer wieder kommt die Sorge auf, dass die Form der Bewertung einen negativen Effekt auf die Erreichung der Bildungsziele hat (auch im deutschen Diskurs oft als Washback oder Backwash bezeichnet). Im Fall eines TraPS muss darüber hinaus vermieden werden, dass negative Rückwirkungen gegenüber den Praxis- und Forschungszielen auftreten. Es gilt, folgende Kriterien zu erfüllen:

1) Bildungs-, Forschungs- und Praxisziele werden gleichermaßen in der Bewertung abgebildet.

2) Die Bewertungsgrundlage verursacht möglichst wenig zusätzlichen Aufwand für die Lernenden.

3) Die Bewertung tariert die Gruppenleistung (in den Studierendenteams) und die individuelle Leistung (die Unterschiede im Eingangsniveau berücksichtigen kann) aus.

In einem TraPS werden, um diesen Anforderungen gerecht zu werden, zwei oder drei gleich gewichtete Leistungen erbracht (s. Tab. 6):

- In einem Lernportfolio ordnen Student(inn)en jeweils individuell alle Dokumente ein, die sie im Seminarverlauf bearbeitet bzw. erstellt haben: Fragen und Kommentare zu einführenden Texten, Projektideen, die Projektskizze, Rechercheergebnisse, Kurzinterviews, Protokolle von Treffen und individuelle Beiträge zum Ergebnis. Diese Texte (und Skizzen etc.) entstehen ohnehin im Arbeitsprozess, ihre systematische Sammlung stellt nur einen geringen Mehraufwand dar. Nur zwei Dokumente werden spezifisch für das Lernportfolio erstellt: Die Reflexion der eigenen Lernziele zu Beginn (Phase II) und die Abschlussreflexion (Phase V). Insgesamt dient das Lernportfolio primär dazu, dass die Student(inn)en ihren individuellen Bildungsprozess kontinuierlich dokumentieren und reflektieren. Lernportfolios, obwohl vielen Lehrenden unbekannt, stellen inzwischen eine recht anerkannte Prüfungsform dar, so dass bei Bedarf hierzu hochschuldidaktische Beratung an fast jeder Hochschule verfügbar sein sollte. Wesentliche Kriterien zur Bewertung sind die vollständige Abbildung des Projekts, die Dokumentation relevanter Projektbeiträge und die Tiefe der Abschlussreflexion.

- Das Resultat, inklusive der Präsentation, als Gruppenleistung. Wenn das Projektergebnis nicht selbst textförmig ist, sondern etwa ein Film, wird ein 
Tabelle 6: Passung der Studienleistungen im TraPS zu den Zieldimensionen.

\begin{tabular}{|l|l|l|}
\hline Bewertete Studienleistung & Sozialform & passt insbesondere zu \\
\hline Portfolio & Individuell & Bildungsziele \\
\hline Projektergebnis inkl. Präsentation & Team & Praxisziele \\
\hline Hausarbeit (ergänzend) & Individuell oder zu zweit & Forschungsziele \\
\hline
\end{tabular}

kurzes, ausformuliertes Handout mit abgegeben, das auch eine bessere Grundlage zur Bewertung darstellt. Bei der Benotung wird die praktische Wirksamkeit - vorzugsweise beurteilt von den Praxispartnern - mit einbezogen. Für diese Leistung sollte man von vorneherein anbieten, $50 \%$ der Note durch individuelle mündliche Prüfungen zu ersetzen, beispielsweise falls ein Gruppenprozess scheitert. In den sechs TraPS in Karlsruhe gab es keinen einzigen Fall, in dem Student(inn)en davon tatsächlich Gebrauch machten. Allein diese Option zu haben, hat aber mehrfach Studierendenteams geholfen, entspannter mit Konflikten umzugehen.

- Für Student(inn)en, die die maximale Zahl an Leistungspunkten (LP) erwerben wollen (in Karlsruhe: 6 statt 4 LP), besteht die Möglichkeit, eine (individuelle) Hausarbeit zu erstellen. Diese darf auf der eigenen Projektarbeit aufbauen, und sie muss nicht zwingend für die Praxispartner relevant sein, sondern soll primär wissenschaftlich gehaltvoll und interessant für die Student(inn)en sein.

Dieser Bewertungsansatz erlaubt es, einer äußerst heterogenen Gruppe von Student(inn)en aus allen Studiengängen, im Bachelor- oder Masterstudium, die unterschiedliche (benotete oder unbenotete) Scheine benötigen, gerecht zu werden. Je nach der Einbettung des TraPS in Studiengänge und fachübergreifende Programme muss dieses Bewertungssystem so angepasst werden, dass die drei eingangs im Abschnitt genannten Punkte (1.-3.) erreicht werden. Selbstverständlich muss es den Student(inn)en von Beginn an transparent gemacht werden.

\subsection{Das Sokratische Gespräch}

Das Sokratische Gespräch ist eine Unterrichtsmethode, die sich aus der philosophischen Tradition des dialogischen Argumentierens ableitet. Während in der Philosophie zumeist nur zwei Personen beteiligt sind, eignet es sich als didaktische Methode auch für Gruppen (Raupach-Strey 2002). Im Sokratischen Gespräch kommt einer Person - im Fall der TraPS einem Mitglied des Lehrteams die Rolle zu, durch systematisches Fragen anderen, insbesondere Student(inn)en, bei der Entwicklung ihrer eigenen Gedanken zu helfen. Die Fragen werden dabei so gestellt, dass die fragende Person keine eigenen Ideen einbringt und die Be- 
fragten inhaltlich nicht leitet. Die Fragen dienen vielmehr den Befragten dazu, ihre eigenen Überlegungen zu vertiefen, weiterzutreiben, Widersprüche zu entdecken oder, im Fall einer Gruppe, sich zu verständigen. Bei der Arbeit mit einer Gruppe können sich dabei Fragen, wie sie auch in der Moderation üblich sind („Sehen Sie das auch so wie X?" „Haben Sie verstanden, was Y meint?“), abwechseln mit solchen, die zu einem tieferen Verständigungsprozess hinleiten („Was meinen Sie mit ,nützlich“?" „Wenn Sie beide von ,Vertrauen“ sprecht, meinen Sie dasselbe - wer vertraut wem?"). Das Sokratische Gespräch unterstützt auf diese Weise analytisches und kritisch-reflexives Denken sowie Kreativität (s. Stenning et al. 2016). Es eignet sich insbesondere dazu, einen spezifischen Fall im Licht von theoretischen Konzepten zu diskutieren (z. B. in der genetischexemplarisch-sokratischen Methode, s. Wagenschein 1999).

Das Sokratische Gespräch muss im Rahmen eines TraPS umsichtig eingesetzt werden, da unterschiedliche Rollenerwartungen an das Lehrteam gestellt werden: Wenn dieses etwa in seiner Expertenrolle angesprochen wird oder weil es verantwortlich für den Rahmen ist, kann sokratisches Nachfragen statt klarer Ansagen und Auskünfte schnell zu Irritationen führen. Nichtsdestotrotz ist das Sokratische Gespräch ein wichtiges Mittel, um die Studierendenteams zu unterstützen, aber zugleich das jeweilige Projekt in deren Verantwortung zu lassen.

\subsection{Die Gestaltung der Lernumgebung}

Sofern die Möglichkeit dazu besteht, sollte die Lernumgebung (Learning Environment, s. Jonassen und Land 2012) für ein TraPS passend zu den spezifischen Arbeitsformen vorbereitet werden. Dies hat eine offensichtliche praktische und eine subtile Seite.

Praktisch gilt es, die Räume für die unterschiedlichen Arbeitsformen im Seminar auszustatten: Je nach Phase und Arbeitsschritt werden ein Plenum, Gruppentische, Platz für flüsternde Zweiergespräche oder individuelle Stillarbeit benötigt. Teilweise sind Visualisierungen (Beamer, Flipchart) nötig, teilweise würden sie ablenken. Laptops, Tablets und Smartphones sind zur Recherche unerlässlich, stören aber in anderen Arbeitsschritten. Alle Materialien, die zuvor im TraPS erarbeitet wurden, sollten jederzeit wieder greifbar sein (typischerweise z. B. Flipchartblätter, Karten). Es lohnt sich, Stauraum dafür vorzusehen und auf systematische Beschriftungen zu achten. Je nach Projekt der Student(inn)en besteht auch noch weiterer Bedarf nach Infrastruktur (z. B. Zugriff auf einen Drucker), auf den die Lernumgebung möglichst flexibel ausgerichtet sein sollte. Wenn ein Programm zur virtuellen Unterstützung der Kooperation im Seminar eingesetzt wird, sollte auch dieses auf seine Tauglichkeit als Lernumgebung hin überprüft und gegebenenfalls gestaltet werden. In den Karlsruher TraPS fungierte der Zukunftsraum im Reallabor Karlsruhe als Lernumgebung (s. Anhang zu diesem Beitrag). 
Subtiler ist die Bedeutung des Raums für die Arbeitsatmosphäre, für den ,Stil ‘ der Veranstaltung. TraPS können, ob geplant oder nicht, ,unter die Haut gehen', unbewusste Selbstverständlichkeiten, Gewohnheiten und Ansichten zum Vorschein bringen, herausfordern oder auch schlummernden Veränderungswillen anstoßen. Im Gruppenprozess gilt es, vermeintlich dumme Nachfragen, Kritik, das Erkennen von Irrwegen in der Projektarbeit und die Bearbeitung von Spannungen zu ermöglichen. Je näher den Student(inn)en (sowie dem Lehrteam und den Praxispartnern) die Themen liegen, desto mehr kann die Arbeit deren Selbstverständnis berühren, desto sensibler sind auch die Gruppenprozesse. Es ist ein wichtiger Teil der Lehraufgaben im TraPS, hierfür einen geschützten Rahmen zu bieten. Der physische Raum, in dem dies alles stattfindet, sollte (gleichermaßen wie die Haltung des Lehrteams) diesem Bedarf Rechnung tragen. Er sollte offen, inspirierend und einladend sein, zugleich aber auch genug Ruhe und Ernsthaftigkeit bieten, um solche Prozesse ungestört gestalten zu können. Das Lehrteam (und evtl. die Praxispartner) sollten sich im physischen Raum möglichst ,zu Hause' fühlen und mit geübter Selbstverständlichkeit alle Möglichkeiten des Raumes nutzen können.

\section{Reflexion}

Die Erfahrung mit den TraPS (s. Anhang) wird hier zunächst anhand von zwei zentralen Aspekten reflektiert, die von allgemeiner Relevanz sind: Zum Ersten anhand des Umgangs mit Zielkonflikten (s. Abschnitt 5.1), zum Zweiten anhand des Umgang mit Erwartungen und Trägerschaft (s. Abschnitt 5.2). Beide bergen das Risiko, Spannungen im Gruppenprozess hervorzurufen, lassen sich aber nicht allein auf der Ebene der Gruppendynamik bearbeiten, da sie eng mit den Inhalten, dem transformativen Anspruch und der transdisziplinären Arbeitsweise verbunden sind. Abschließend wird die Erfahrung mit der spezifischen Situation, TraPS in einem Reallabor zu verankern, dargestellt (s. Abschnitt 5.3).

\subsection{Vorausschauender und entspannter Umgang mit Zielkonflikten}

Ein TraPS dient dazu, Ziele in drei Dimensionen zu verfolgen, Forschungs-, Praxis- und Bildungsziele (s. Beecroft et al. 2018) - dieser Zielhorizont kann zu Zielkonflikten führen. Das bedeutet, dass nicht nur verschiedene Beteiligte unterschiedliche Interessen verfolgen (Interessenkonflikte, die es gegebenenfalls auch auszuhandeln und auszuhalten gilt), sondern dass die Beteiligten gemeinsam Ziele in unterschiedlichen Dimensionen anstreben, diese Ziele aber in Konkur- 
renz zueinander geraten können. ${ }^{2}$ Solche Zielkonflikte sollten vorausschauend identifiziert und unaufgeregt thematisiert werden.

Es lohnt sich, die individuellen Ziele aller Beteiligten zu explizieren, um bereits von Anfang an mögliche Konflikte aufzuspüren. Dies geschieht in Phase I mit den Praxispartnern, in den Phasen II und III mit den Student(inn)en, die gemeinsam in den Studierendenteams einen Zielhorizont für ihr jeweiliges Projekt festlegen. Eventuell gelingt es bereits zu diesem frühen Zeitpunkt, eine Handhabe für die Zielkonflikte zu finden, zumindest die Prioritäten der Ziele zu benennen und damit in den Projekten auch zu überprüfen, was essenziell ist.

In einem TraPS ist ein recht entspannter Umgang mit den Zielen ratsam: Nicht jedes Studierendenprojekt wird Ziele in allen drei Dimensionen im selben Maß erreichen können. Wenn von einem Studierendenteam nur in zwei der drei Zieldimensionen Ziele erreicht werden, ist das völlig ausreichend. Gegebenenfalls ist immer noch möglich, durch ein ergänzendes, komplementäres Element (z. B. eine Hausarbeit, einen Workshop, ein separates Dokument) in Phase VI ein Ziel zu verfolgen, das sonst in den Hintergrund gerückt wäre. Schließlich stellt die Fähigkeit, Zielkonflikte zu erkennen und zu bearbeiten, auch eine wichtige Kompetenz dar, die die Student(inn)en in einem TraPS erwerben können - so dass ihre Bearbeitung selbst schon einem Bildungsziel dient.

Positiv gewendet lässt sich sagen, dass ein TraPS als Ansatz gerade auch dann interessant ist, wenn schon die Ausgangsbedarfe des Praxispartners explizit oder implizit Zielkonflikte aufweisen: In solchen Fällen können die parallel arbeitenden Studierendenteams Einzelbeiträge liefern, die zusammengenommen allen Zieldimensionen dienlich sind.

\subsection{Erwartungen managen und Trägerschaft mitdenken}

Der dreifache Zielhorizont kann es mit sich bringen, dass die verschiedenen Beteiligten nicht im Einklang stehende Erwartungen aneinander und an das TraPS insgesamt haben, die gleichzeitig nicht selten diffus und implizit bleiben. In den Karlsruher TraPS hat es sich bewährt, den Zielhorizont und die damit verbundenen Erwartungen sehr explizit zum Thema zu machen, und zwar zunächst in Phase I in einer Diskussion mit den Praxispartnern, in Phase II in einer Diskussion mit den Student(inn)en und indem in Phase III den Studierendenteams dann Gelegenheit gegeben wird, eigene Ansprüche zu formulieren.

2 Natürlich kommt es mitunter auch innerhalb der Forschungs-, Bildungs- und Praxisziele zu Zielkonflikten; in diesen Fällen sind die Ziele aber vergleichbar und eine Abwägung dadurch deutlich leichter möglich. 
Die Erwartungen der Praxispartner sind besonders zu Beginn oft diffus und nicht übermäßig hoch. Die Student(inn)en wiederum bringen zunächst Erwartungen mit ins TraPS, die sich auf den Titel und den Veranstaltungskommentar beziehen. Ungünstige Beschreibungen können von Anfang an falsche Erwartungen wecken. Vorsicht ist auch geboten in Bezug auf Versprechungen, dass das TraPS ,Spaß macht ${ }^{\star}$. Insbesondere in der Projektarbeit kommt es immer wieder zu Situationen, die weniger Spaß machen. Andere Motivationen, etwa die Möglichkeit, eigenen Interessen $\mathrm{zu}$ folgen oder praxisrelevante Arbeitstechniken zu erlernen, sind in dieser Hinsicht robuster.

Bei der Erstellung der Projektskizzen (Phase III) neigen die Student(inn)en zu hohen, teils auch unrealistischen Erwartungen an ihr eigenes Projekt, wobei die Bildungs- und Forschungsziele tendenziell weniger klar gefasst sind als die Praxisziele. Dabei können übersteigerte Erwartungen an die eigene Leistungsfähigkeit, an die Qualität des geplanten Resultats und an dessen Nutzung durch die Praxispartner Hand in Hand gehen. Möglicherweise ist dies bei TraPS im Rahmen eines Fachstudiums anders, da die Student(inn)en dort vielleicht klarere Vorstellungen mitbringen in Bezug auf die eigenen Bildungsziele, den wissenschaftlichen Anspruch des Fachs und den Aufwand der einzusetzenden Methoden. Gelegentlich kann ein Projekt eines Studierendenteams in praktischer Hinsicht gänzlich scheitern; in diesem Fall ist es besonders wichtig, die Erwartungen in den anderen beiden Zieldimensionen im Blick zu behalten. Gegenüber den Praxispartnern sollte die Möglichkeit, dass Projekte in Bezug auf die Praxisziele scheitern können, bereits in Phase I deutlich gemacht werden.

Als besonders relevant haben sich Erwartungen herausgestellt, die die Trägerschaft betreffen, also die mittelfristige Verantwortung für und Verfügung über die Resultate: Jedes Projekt eines Studierendenteams ist verbunden mit einer Vorstellung darüber, wer in Folge was damit tun soll. Manche Ergebnisse können ohne Aufwand genutzt werden (z. B. ein Infoposter), andere erfordern aber intensive Beschäftigung und eine klare Umsetzungsabsicht (z. B. ein Planspiel). Wenn die Trägerschaft nicht schon während der Erstellung der Projektskizzen mitbedacht und in der Projektarbeit verbindlich gemacht wird, kann es nach der Übergabe der Ergebnisse zu Enttäuschungen seitens der Student(inn)en kommen. Genauso wie die Praxispartner ihnen den Freiraum zugestehen müssen, die eigenen Projekte zu skizzieren, müssen die Studierendenteams ihr Projektergebnis am Ende loslassen. Die explizite Diskussion und Reflexion der Ziele und Erwartungen aller Beteiligten kann solchen Enttäuschungen entgegenwirken und die Sensibilität dafür erhöhen, welche Akteure welche Art Freiheit und Autonomie brauchen. Gerade in der Kooperation mit kleinen zivilgesellschaftlichen Initiativen muss mit der Erwartung, dass diese die Trägerschaft übernehmen, vorsichtig umgegangen werden: Die Student(inn)en können im Extremfall gemeinsam über 4.000 Arbeitsstunden (bei 6 ECTS und 24 Student(inn)en) in die Projekte investieren - eine kleine Initiative kann nur einen Bruchteil dessen für die langfristige Trägerschaft aufwenden. 


\subsection{Besonderheiten der Einbettung eines TraPS in einem Reallabor}

In Karlsruhe waren die TraPS in ein Reallabor eingebettet (s. Abschnitt 1.1). Auch wenn diese Einbettung nicht notwendig ist für ein TraPS, bietet ein Reallabor doch eine wertvolle Unterstützungsstruktur, von der vielleicht einzelne Elemente in andere Projektrahmen übertragen werden können. Da ein Reallabor selbst Forschungs-, Praxis- und Bildungsziele verfolgt, ist die Passung zu einem TraPS leicht herzustellen. Die in Beecroft et al. (2018) skizzierten Designprinzipien können beispielsweise herangezogen werden, um die Passung zwischen dem Reallabor - oder einem vergleichbaren Vorhaben im Grenzbereich von Wissenschaft und Praxis - und dem TraPS zu optimieren. Dabei gilt es zu bedenken, dass ein TraPS darauf ausgelegt ist, von einer Rahmung zu profitieren, die transformative Prozesse unterstützt, und umgekehrt selbst zu einer solchen Rahmung beizutragen.

Erstens stellt ein Reallabor einen Forschungskontext dar, in dem transdisziplinäre Projekte stattfinden. Es ist möglich, einzelne Studierendenprojekte im TraPS an übergreifende Themen und Fragen im Reallabor anzuschließen und im TraPS entsprechende Theoriebezüge, Evaluationsmechanismen, eine Dokumentationsinfrastruktur usw. zu nutzen. Das interdisziplinäre Reallaborteam wiederum stellt eine wichtige Ressource für das TraPS dar, wenn weitere Expert(inn)en zu Teilfragen oder spezifischen Methoden gesucht werden. Das TraPS seinerseits kann zur Forschung im Reallabor beitragen, indem entweder eine systematische Integration von Fallstudien im Reallabor angelegt ist oder auch Forschungsfragen in das TraPS bereits in der Konzeption mit eingespeist werden.

Zweitens bietet das Reallabor mit den von ihm gepflegten Kooperationen mit Praxisakteuren einen Praxiskontext für das TraPS. Das Reallabor zielt darauf ab, Transformationsprozesse zu initiieren und zu begleiten. In diesem Kontext können auch die Praxisziele eines TraPS verfolgt werden. Das Reallabor unterhält ein Netzwerk von Akteuren, aus dem sich Praxispartner für ein TraPS gewinnen lassen. Das TraPS kann umgekehrt dazu eingesetzt werden, bestehende Kooperationen für ein Reallabor zu vertiefen.

Drittens stellt das Reallabor eine Lernumgebung dar. Diese umfasst die physische und virtuelle Infrastruktur des Reallabors, die Räume, Materialien, Arbeitsplätze etc., aber ebenso den Untersuchungsraum des Reallabor, über den im Reallabor bereits ein erheblicher Wissensschatz zur Verfügung steht. Da das Reallabor darauf ausgerichtet ist, iterative Lernprozesse zu ermöglichen, können umgekehrt die Erfahrungen aus einem TraPS in die Weiterentwicklung des Reallabors selbst und in die Lernprozesse aller Beteiligten einfließen. In Form eines TraPS kann universitäre Lehre in gesellschaftliche Lernprozesse eingebettet werden - zum beiderseitigen Vorteil. 


\section{Dank}

Der Autor dankt Charlotte Eller für die Diskussionen über eine frühere Textfassung. Insbesondere möchte er Marius Albiez, Andri König, Astrid Ley und Thomas Potthast sowie zwei anonymen Gutachter(inne)n für die konkreten Hinweise zur Verbesserung des Textes im Rahmen des internen und externen Reviews danken. Schließlich dankt der Autor den beiden Herausgebenden, Rico Defila und Antonietta Di Giulio, für ihre Rückmeldungen zum Text.

\section{Literatur}

Beecroft, R., \& Parodi, O. (2016). Reallabore als Orte der Nachhaltigkeitsforschung und Transformation. Einführung in den Schwerpunkt. Technikfolgenabschätzung - Theorie und Praxis, 25 (3), (S. 4-8).

Beecroft, R., Trenks, H., Rhodius, R., Benighaus, C., \& Parodi, O. (2018). Reallabore als Rahmen transformativer und transdisziplinärer Forschung: Ziele und Designprinzipien. In R. Defila \& A. Di Giulio (Hrsg.), Transdisziplinär und transformativ forschen. Eine Methodensammlung (S. 75-100). Wiesbaden: Springer VS. doi: 10.1007/978-3-65821530-9_4.

Beecroft, R. (2018). Embedding Higher Education into a Real-World Lab: A ProcessOriented Analysis of Six Transdisciplinary Project Courses. Sustainability, 10 (10), (Nr. 3798). doi: 10.3390/su10103798.

DeZure, D. (2010). Interdisciplinary pedagogies in higher education. In R. Frodeman, J. Klein \& R. Pacheco (Hrsg.), The Oxford handbook of interdisciplinarity (S. 372-386). Oxford: Oxford University Press.

Higgins, L., \& Litzenberg, K. (2015). Transferring experience through team teaching: The chance of a lifetime. College teaching, 63 (3), (S. 105-111).

Huber, L. (2009). Warum Forschendes Lernen nötig und möglich ist. In L. Huber, J. Hellmer \& F. Schneider (Hrsg.), Forschendes Lernen im Studium. Aktuelle Konzepte und Erfahrungen (S. 9-35). Bielefeld: Universitäts-Verlag.

Huber, L. (2014). Forschungsbasiertes, Forschungsorientiertes, Forschendes Lernen: Alles dasselbe? Ein Plädoyer für eine Verständigung über Begriffe und Unterscheidungen im Feld forschungsnahen Lehrens und Lernens. Das Hochschulwesen, 62 (1/2), (S. 32-39).

Hurd, C. A. (2008). Is service-learning effective? A look at current research. In S. Shalini (Hrsg.), Service-learning: Perspectives and applications (S. 44-60). Punjagutta: ICFAI University Press. Manuskript vom Juni 2006 verfügbar unter http://fresnostate.edu/ craig/depts-programs $/ \mathrm{mktg} /$ documents/Is $\quad$ S.L. Effective-.pdf. Zugegriffen am 09.03.2019. 
Jonassen, D., \& Land, S. (Hrsg.). (2012). Theoretical foundations of learning environments. Routledge: New York.

Meyer-Soylu, S., Parodi, O., Trenks, H., \& Seebacher, A. (2016). Das Reallabor als Partizipationskontinuum. Erfahrungen aus dem Quartier Zukunft und Reallabor 131 in Karlsruhe. Technikfolgenabschätzung - Theorie und Praxis, 25 (3), (S. 31-40).

Michael, L. (1963). Team teaching. The bulletin of the National Association of Secondary School Principals, 47 (283), (S. 36-63).

Mieg, H., \& Lehmann, J. (Hrsg.). (2017). Forschendes Lernen: Wie die Lehre in Universität und Fachhochschule erneuert werden kann. Frankfurt, New York: Campus.

Murata, R. (2002). What does team teaching mean? A case study of interdisciplinary teaming. The Journal of educational research, 96 (2), (S. 67-77).

Parodi, O., Albiez, M., Beecroft, R., Meyer-Soylu, S., Quint, A., Seebacher, A., Trenks, H., \& Waitz, C. (2016). Das Konzept „Reallabor“ schärfen: Ein Zwischenruf des Reallabor 131: KIT findet Stadt. GAIA, 25 (4), (S. 284-285). doi:10.14512/gaia.25.4.11.

Raupach-Strey, G. (2002). Sokratische Didaktik. Die didaktische Bedeutung der Sokratischen Methode in der Tradition von Leonard Nelson und Gustav Heckmann. Münster: Lit.

Reitinger, J. (2013). Forschendes Lernen. Theorie, Evaluation und Praxis. Theorie und Praxis der Schulpädagogik, Band 12. Immenhausen bei Kassel: Prolog.

Reitinger, J., Haberfellner, Ch., \& Keplinger, G. (2016). An Overview of the Theory of Inquiry Learning Arrangements (TILA). In J. Reitinger, C. Haberfellner, E. Brewster \& M. Kramer (Hrsg.). Theory of inquiry learning arrangements: research, reflection, and implementation (S. 1-11). Kassel: Universitätsverlag Kassel.

Rueß, J., Gess, C., \& Deicke, W. (2016). Forschendes Lernen und forschungsbezogene Lehre - empirisch gestützte Systematisierung des Forschungsbezugs hochschulischer Lehre. Zeitschrift für Hochschulentwicklung, 11 (2), (S. 23-44).

Seifert, A., Zentner, S., \& Nagy, F. (2012). Praxisbuch Service-Learning: „Lernen durch Engagement" an Schulen. Mit Materialien für Grundschule und Sekundarstufe I + II. Weinheim: Beltz.

Stenning, K., Schmoelz, A., Wren, H., Stouraitis, E., Scaltsas, T., Alexopoulos, C., \& Aichhorn, A. (2016). Socratic dialogue as a teaching and research method for cocreativity? Digital Culture \& Education, 8 (2), (S. 154-168).

Trenks, H., Waitz, C., Meyer-Soylu, S., \& Parodi, O. (2018). Mit einer Realexperimentreihe Impulse für soziale Innovationen setzen - Realexperimente initiieren, begleiten und beforschen. In R. Defila \& A. Di Giulio (Hrsg.), Transdisziplinär und transformativ forschen. Eine Methodensammlung (S. 233-268). Wiesbaden: Springer VS. doi: 10.1007/978-3-658-21530-9_12. 
Vilsmaier, U., \& Meyer, E. (2017). Forschendes Lernen in der Nachhaltigkeitswissenschaft. In H. Mieg \& J. Lehmann (Hrsg.). Forschendes Lernen: Wie die Lehre in Universität und Fachhochschule erneuert werden kann (S. 357-366). Frankfurt/New York: Campus.

Wagenschein, M. (1999). Verstehen lehren: genetisch, sokratisch, exemplarisch. Weinheim: Beltz.

Waitz, C., Quint, A., Trenks, H., Lezuo, D., Jäkel, A., Wäsche, H., \& Parodi, O. (2018). Das Reallabor als Motor für nachhaltige Quartiersentwicklung - Erfahrungen aus dem Karlsruher Experimentierraum. Berichte. Geographie und Landeskunde, 91 (1), (S. 67-80).

Wals, A. (Hrsg.). (2007). Social learning towards a sustainable world: Principles, perspectives, and praxis. Wageningen: Wageningen Academic Pub.

Welch, M., Brownell, K., \& Sheridan, S. (1999). What's the score and game plan on teaming in schools? A review of the literature on team teaching and school-based problemsolving teams. Remedial and Special Education, 20 (1), (S. 36-49). 


\section{Anhang}

\section{Die Erfahrungen mit sechs TraPS in Karlsruhe}

Die untenstehende Tabelle gibt eine Übersicht über die sechs TraPS, die in Karlsruhe innerhalb von drei Jahren durchgeführt wurden. Die Teilnehmerzahl spiegelt die Anzahl der Student(inn)en, die bis zum Ende des TraPS beteiligt blieben. Da die Teilnahme freiwillig war, gab es immer wieder Teilnehmerschwund, insbesondere in den ersten Wochen, was auch auf die Kollision mit Pflichtveranstaltungen zurückzuführen war, deren Termine zuerst noch nicht feststanden. Die folgende Darstellung ist eine Übersetzung und Zusammenfassung einer qualitativen Analyse der sechs TraPS (Beecroft 2018, S. 11-14).

Das erste TraPS - „Besser Altern in Karlsruhe“ - wurde gemeinsam mit einem frühen Praxispartner des Reallabors Karlsruhe, einer Gemeindeinitiative zur Verbesserung der Lebensbedingungen in einer benachbarten Gemeinde, und mit sehr wenigen Student(inn)en durchgeführt. Nach den ersten Wochen veränderte sich die Kooperation im Kurs wesentlich, als die Praxispartner ein Thema einbrachten, zu dem sie Unterstützung suchten (die Planung und Durchführung einer Infoveranstaltung zu Notknöpfen). Mit diesem Bedarf waren die Praxisziele gesetzt und es entwickelte sich im Wesentlichen eine Service-Learning-Dynamik. Die Student(inn)en fanden einen weiteren Praxisakteur (einen Anbieter von Notknöpfen), die

Tabelle: Die sechs Karlsruher TraPS.

\begin{tabular}{|c|c|c|c|c|}
\hline Semester & Titel & Veranstalter & Praxispartner & TN \\
\hline $\begin{array}{l}\text { WS } \\
2014 / 15\end{array}$ & Besser Altern in Karlsruhe & $\begin{array}{l}\text { Beecroft, } \\
\text { Albiez }\end{array}$ & $\begin{array}{l}\text { Gemeindeinitia- } \\
\text { tive Gut Altern }\end{array}$ & 3 \\
\hline $\begin{array}{l}\text { SS } \\
2015\end{array}$ & Zu Tisch! & $\begin{array}{l}\text { Albiez, } \\
\text { Beecroft }\end{array}$ & $\begin{array}{l}\text { Karlsruher Tafel } \\
\text { e. V. }\end{array}$ & 7 \\
\hline $\begin{array}{l}\text { WS } \\
2015 / 16\end{array}$ & $\begin{array}{l}\text { Reparieren, Wieder- } \\
\text { verwerten, Selbstmachen! }\end{array}$ & $\begin{array}{l}\text { Tamm, } \\
\text { Beecroft }\end{array}$ & $\begin{array}{l}\text { ReparaturCafé } \\
\text { Initiative Karls- } \\
\text { ruhe }\end{array}$ & 14 \\
\hline $\begin{array}{l}\text { SS } \\
2016\end{array}$ & $\begin{array}{l}\text { Gemeinwohlökonomie für } \\
\text { Karlsruhe? }\end{array}$ & $\begin{array}{l}\text { Beecroft, } \\
\text { Tamm }\end{array}$ & $\begin{array}{l}\text { GWÖ-Initiative } \\
\text { Karlsruhe }\end{array}$ & 12 \\
\hline $\begin{array}{l}\text { WS } \\
\text { 2016/17 }\end{array}$ & $\begin{array}{l}\text { Bildung für Nachhaltige } \\
\text { Entwicklung planen }\end{array}$ & $\begin{array}{l}\text { Beecroft, } \\
\text { Friedrichs }\end{array}$ & ZAK, KIT & 3 \\
\hline $\begin{array}{l}\text { SS } \\
2017\end{array}$ & $\begin{array}{l}\text { Nachhaltigkeitsspaziergang } \\
\text { Karlsruhe }\end{array}$ & $\begin{array}{l}\text { Tamm, } \\
\text { Beecroft }\end{array}$ & $\begin{array}{l}\text { stattreisen e. V. } \\
\text { Stadt Karlsruhe }\end{array}$ & 16 \\
\hline
\end{tabular}


Praxispartner warben intensiv in ihrem Umfeld für die Infoveranstaltung, mit mäßigem Erfolg, und die Student(inn)en betteten das vorgegebene Thema in einen weiteren Kontext ein. Es war essenziell, mit den Student(inn)en ein Nachtreffen ohne die Praxispartner durchzuführen, um die Rollen und den Seminarverlauf zu reflektieren. Dieser Kurs war der ,Prototyp ' für die hier vorgestellte Struktur, die erst in Folge formalisiert und weiterentwickelt wurde.

Das zweite TraPS - „Zu Tisch!“ - in Kooperation mit einer der Karlsruher Tafeln fokussierte auf die sozialen Fragen nachhaltiger Ernährung. Nach einem Einstieg, in dem herausgearbeitet werden musste, dass die Veranstaltung kein Rekrutierungsevent für den Praxispartner ist, starteten die Student(inn)en eine kritische Diskussion über die Angemessenheit von Tafeln. Diese konnte in die beiden Studierendenprojekte teilweise einbezogen werden, die sich auf Informationen zur Nahrungsmittellagerung für die Kundschaft und das Abfallmanagement der Tafel bezogen. Letzteres stellte sich als analytisch anspruchsvolles Thema heraus, führte aber zu dem unspektakulären Ergebnis, dass das bestehende System nahezu ideal sei. Die Praxispartner blieben zurückhaltend, die Student(inn)en im laufenden Betrieb in die Tafel einzuladen, um keine Irritation bei der Kundschaft hervorzurufen. Die (hochschulweit standardisierte) Evaluation der Veranstaltung machte mit einem kritischen Ergebnis die Orientierungslosigkeit deutlich, die zu Beginn der Projektphase eingetreten war. Eine Reflexion mit den Student(inn)en am Ende des TraPS war wesentlich, um unausgesprochene Erwartungen (z. B. bezüglich der Rolle der Lehrenden), Fragen der Gerechtigkeit bei sehr unterschiedlichen Projekten und Zielkonflikte zwischen praktischer Wirksamkeit und wissenschaftlicher Relevanz aufzuarbeiten. Die Erfahrung mit diesem Kurs führte zu einer systematischen Explikation der Forschungs-, Bildungs- und Praxisziele zu Beginn der Veranstaltung und zur Einführung eines Probelaufs der Präsentation.

Das dritte TraPS - „Reparieren, Wiederverwerten, Selbstmachen!“ - mit einem Fokus auf Konsum und Nachhaltigkeit unterstützte die ReparaturCafé-Initiative in Karlsruhe, die selbst aus einem Event des Reallabors hervorgegangen war und dann eng mit dem Reallabor kooperierte. Zum Zeitpunkt des Kurses war die Initiative dabei, einen eigenständigen eingetragenen Verein zu gründen. Die ursprüngliche Hoffnung des Praxispartners, insbesondere zu Versicherungs- und Haftungsfragen Unterstützung zu erhalten, musste enttäuscht werden, da die Student(inn)en keinerlei einschlägige Kenntnisse hatten. Nichtsdestotrotz konnten die vier Studierendenteams mehrere wertvolle Beiträge liefern: einen Imagefilm, um auch online Sichtbarkeit für die Initiative und ihre konsumkritischen Themen herzustellen, ein Planspiel zum Test der geplanten Vereinssatzung, ein Flugblatt mit Hinweisen zu Fachhändlern im Quartier, die Ersatzteile verkaufen, sowie mehrere analytische Beiträge zu Kooperationspotenzialen und zur Vergleichbarkeit mit anderen Initiativen. Die vielfältigen Ergebnisse waren für den Praxis- 
partner nur knapp zu bewältigen, dementsprechend wurden Fragen der Trägerschaft seit diesem Kurs in das Konzept eines TraPS aufgenommen. Es war der erste Kurs, der den frisch eingeweihten „Zukunftsraum“ als Lernumgebung nutzen konnte. In diesem Kurs fand die Ausdifferenzierung der Studienleistungen die in diesem Beitrag vorgestellte Form, und die Vorstellung der Projektskizzen erfolgte erstmals systematisch.

Das vierte TraPS - „Gemeinwohlökonomie (GWÖ) für Karlsruhe?“ - mit der lokalen GWÖ-Initiative thematisierte nachhaltiges Wirtschaften. Mit einem CoLehrenden aus der GWÖ-Initiative und einer Reihe von weiteren Mitgliedern der Initiative, die bei mehreren Seminarterminen präsent waren, bildete sich eine enge Zusammenarbeit heraus. Die dadurch verursachte Vermutung der Student(inn)en, von ihnen würde erwartet, die Position der GWÖ zu nachhaltigem Wirtschaften ohne Kritik zu übernehmen, musste direkt angesprochen und ausgeräumt werden. Die kritischen Fragen konnten so in zwei der vier Studierendenprojekte einbezogen werden, was zu mehr Tiefe in der Auseinandersetzung mit den Projektthemen führte. Als lokaler Zweig eines aktiven internationalen Netzwerks konnten die Praxispartner weitere Spezialist(inn)en aus dem GWÖ-Netzwerk einbeziehen, und auch die Ergebnisse konnten so weiter verbreitet werden. Eine Studierendengruppe erstellte eine erste (minimale) GWÖ-Bilanz eines Vereins und konnte so Unterschiede zum Einsatz in Unternehmen ausweisen. Ein Informationsfilm zur GWÖ wurde - Jahre später - eine der meistgenutzten deutschsprachigen Online-Ressourcen zur Information über den Ansatz. In diesem Kurs wurde die Verwendung eines Online-Projekttools getestet, mit dem die Praxispartner arbeiten - dieses wurde aber nicht als fester Baustein in das Seminarkonzept aufgenommen, da die Studierendenprojekte zu unterschiedlich gelagert waren für ein einheitliches System. An diesem Kurs wurden die Überlegungen zur langfristigen Trägerschaft verfeinert und die Rollenklärungen mit den Praxispartnern systematisiert.

Das fünfte TraPS - „Bildung für Nachhaltige Entwicklung planen“ - mit einer kleinen Gruppe von Student(inn)en stellte einen Planungsprozess für eine große Einführungsveranstaltung zu Nachhaltiger Entwicklung dar, in Kooperation mit dem Zentrum für Angewandte Kulturwissenschaft und Studium Generale (ZAK). $\mathrm{Da}$ diese Veranstaltung (die inzwischen unter dem Namen „Frühlingstage der Nachhaltigkeit" auf rund 300 Teilnehmer(innen) ausgelegt ist) von Anfang an als Kooperation mit dem ZAK geplant war, unterschieden die Student(inn)en nicht zwischen den Rollen als Lehrende und als Praxispartner. Dies belastete den Kurs nicht, es mussten aber immer wieder Fragen nach der Offenheit des Prozesses und nach dem Verhältnis zwischen den Bildungs- und Praxiszielen angesprochen und geklärt werden. Dieser Kurs profitierte sehr von den Kompetenzen der Mitglieder des Reallaborteams, die nicht im Lehrteam waren, und vom weiteren wissenschaftlichen Netzwerk des Reallabors. 
Das sechste TraPS - „Nachhaltigkeitsspaziergang Karlsruhe“ - war eingebettet in ein internationales Vergleichsprojekt zu Stadt-Universitäts-Kooperationen. Als Praxispartner waren Vertreter(innen) von fünf Ämtern der Stadt Karlsruhe sowie eines nichtkommerziellen Anbieters von Stadtführungen (Verein) beteiligt. Ein ehemaliger Praktikant des Reallabors führte als Masterarbeit an einer anderen Universität eine Begleitforschung durch. Das Seminar hatte statt einer Skizzenund einer Projektphase zwei Projektphasen: In der ersten gruppierten sich die Student(inn)en zu übergreifenden Fragen (z. B. Zielgruppe, mögliche Route, Nachhaltigkeitstheorien). Nach einer Entscheidung über diese allgemeinen Fragen gemeinsam mit den Praxispartnern arbeiteten die Student(inn)en in neu gemischten Teams Informationen zu einzelnen Stationen aus und bezogen diese auf die Nachhaltigkeitsziele der Vereinten Nationen. Im Projektverlauf wurde die Rolle des zivilgesellschaftlichen Vereins immer aktiver, so dass dieser die Trägerschaft für eines der Produkte, eine geführte Nachhaltigkeitstour, übernahm. Zugleich engagierten sich dann zwei der Student(inn)en dort als Reiseführer. Die Phase der In-Wert-Setzung war, da mehrere parallele Produkte (Audioguide, Online-Karte, Broschüre, geführte Tour) entwickelt wurden, besonders aufwendig und dauerte, auch durch technische Verzögerungen bedingt, lange an. Ein Teil der Ergebnisse wurde zum Teil der Infrastruktur des Reallabors. Das Seminar hatte eine komplexe Architektur, die nicht 1:1 in das in diesem Beitrag vorgestellte Vorgehen für ein TraPS übernommen wurde, da sie kaum ohne ein Reallabor als unterstützenden Rahmen realisierbar ist. In diesem Kurs war erstmals die vielfältige Einbettung ins Reallabor (lokale Netzwerke ausbauen, Infrastruktur entwickeln, Sichtbarkeit erhöhen, wissenschaftliche Vernetzung fördern u. a.) von Anfang an Teil der Detailplanung des TraPS. Durch die Einbettung in ein internationales Vergleichsprojekt wurde die theoretische Aufarbeitung des transdisziplinären Charakters des Seminarablaufs und des transformativen Anspruchs deutlich vorangebracht.

Open Access Dieses Kapitel wird unter der Creative Commons Namensnennung 4.0 International Lizenz (http://creativecommons.org/licenses/by/4.0/deed.de) veröffentlicht, welche die Nutzung, Vervielfältigung, Bearbeitung, Verbreitung und Wiedergabe in jeglichem Medium und Format erlaubt, sofern Sie den/die ursprünglichen Autor(en) und die Quelle ordnungsgemäß nennen, einen Link zur Creative Commons Lizenz beifügen und angeben, ob Änderungen vorgenommen wurden.

Die in diesem Kapitel enthaltenen Bilder und sonstiges Drittmaterial unterliegen ebenfalls der genannten Creative Commons Lizenz, sofern sich aus der Abbildungslegende nichts anderes ergibt. Sofern das betreffende Material nicht unter der genannten Creative Commons Lizenz steht und die betreffende Handlung nicht nach gesetzlichen Vorschriften erlaubt ist, ist für die oben aufgeführten Weiterverwendungen des Materials die Einwilligung des jeweiligen Rechteinhabers einzuholen.

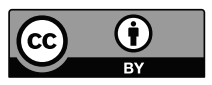

